\title{
Ciliary Motility: Regulation of Axonemal Dynein Motors
}

\author{
Rasagnya Viswanadha, ${ }^{1}$ Winfield S. Sale, ${ }^{1}$ and Mary E. Porter ${ }^{2}$ \\ ${ }^{1}$ Department of Cell Biology, Emory University School of Medicine, Atlanta, Georgia 30322 \\ ${ }^{2}$ Department of Genetics, Cell Biology and Development, University of Minnesota, Minneapolis, Minnesota 55455 \\ Correspondence: porte001@umn.edu
}

\section{SUMMARY}

Ciliary motility is crucial for the development and health of many organisms. Motility depends on the coordinated activity of multiple dynein motors arranged in a precise pattern on the outer doublet microtubules. Although significant progress has been made in elucidating the composition and organization of the dyneins, a comprehensive understanding of dynein regulation is lacking. Here, we focus on two conserved signaling complexes located at the base of the radial spokes. These include the I1/ $f$ inner dynein arm associated with radial spoke 1 and the calmodulin- and spoke-associated complex and the nexin-dynein regulatory complex associated with radial spoke 2. Current research is focused on understanding how these two axonemal hubs coordinate and regulate the dynein motors and ciliary motility.

\section{Outline}

1 Introduction

2 Axonemal structure and a sliding microtubule model for ciliary bending

3 Regulation of axonemal bending by I1/f dynein and the MIA complex located near RS1
4 The RS2 regulatory complex and downstream regulation

5 Conclusion

References 


\section{INTRODUCTION}

Cilia and flagella are highly conserved organelles required for cell signaling and cell motility in many organisms. They are generally classified either as immotile (including primary and sensory cilia) or as motile structures and play essential roles in vertebrate development and function of organs in the adult. We focus here on the structure and mechanisms of motile cilia that, in humans, are essential for embryonic development, sperm motility, and movement of fluid in the airway, oviducts, and brain ventricles. Ciliary motility is driven by the coordinated activity of the dynein motors. However, researchers are only beginning to understand the mechanisms that coordinate and control the dyneins. Here, we consider recent advances in genetic, cell-biological, and structural studies of ciliary dyneins and associated structures in Chlamydomonas. These studies have revealed new insights into the detailed organization of, and the physical interactions among, the dyneins.

In addition, we emphasize the organization of the outer doublet microtubule "96-nm repeat" and discuss two regulatory hubs required for control of ciliary motility: (1) radial spoke 1 (RS1) and its association with an inner dynein arm (IDA) called I1 dynein located near the proximal end of the 96-nm repeat, and (2) radial spoke 2 (RS2) and associated calmodulin- and spoke-associated complex (CSC) and the nexin-dynein regulatory complex (N-DRC) located at the distal end of the 96-nm repeat. Together, these two hubs are thought to coordinate dynein activity and regulate the ciliary waveform and beat frequency. These complexes are also important for modification of motility in response to external and internal signals. Other important topics not discussed here, but covered elsewhere, include the structure and function of the central pair microtubules (Smith and Yang 2004; Lechtreck and Witman 2007; O’Toole et al. 2012; Carbajal-Gonzalez et al. 2013; Oda et al. 2014a, 2014b), and theoretical models for ciliary bending (Brokaw 1985, 2009; Lindemann and Lesich 2010).

\section{AXONEMAL STRUCTURE AND A SLIDING MICROTUBULE MODEL FOR CILIARY BENDING}

\subsection{Axoneme Structure in Motile Cilia}

Motile cilia typically contain a " $9+2$ " axoneme comprising nine outer doublet microtubules and a central apparatus built on two central pair microtubules and their associated projections (Fig. 1). Advances in electron microscopy (EM) have been essential to our understanding of the structure of the axoneme and the mechanism of mo- tility in cilia. Key technical advances include computational approaches and image averaging (Kamiya et al. 1991; Mastronarde et al. 1992; Porter et al. 1992), rapid-freeze, deep-etch rotary-shadow EM (Goodenough and Heuser 1982; Goodenough and Heuser 1985), and, most recently, cryo-electron tomography (cryo-ET) of axonemes and intact cilia (Nicastro et al. 2006; Bui et al. 2009; Nicastro 2009; Bui and Ishikawa 2013; Oda and Kikkawa 2013). In most motile cilia, the nine outer doublet microtubules are organized with a structural axis that is fixed relative to the bending plane. For example, the inset in Figure 1A illustrates the forward (green circle) and reverse (blue circle) bends of the two flagella seen in a typical forwardswimming Chlamydomonas cell. In the cross section of an axoneme from Chlamydomonas, the bending plane passes between doublet \#1 and doublet \#2 (Fig. 1; and see Hoops and Witman 1983). As discussed below, dyneins present on the doublets on opposite sides of the axonemal axis are thought to be responsible for generating the forward (green) and reverse (blue) bends.

In vitro reactivation studies have revealed that all components necessary for movement are physically built into the axoneme (Gibbons and Gibbons 1972). Thus, study of isolated axonemes can reveal many of the features required for regulation of motility. Chlamydomonas has been a particularly powerful model organism for discovery of conserved genes required for ciliary assembly and motility because of its tractable genetics, informative mutant phenotypes, and the ease of culture for biochemical and structural studies (Harris 2009; Dutcher 2014; Kamiya and Yagi 2014).

\subsection{Outer Doublet Microtubule Organization and Heterogeneity of Inner Dynein Arms}

The focus of this review is on the structures of the outer doublet microtubules required to control the dynein motors (Figs. 1 and 2). Outer doublet components are organized in an axonemal 96-nm repeat and include the radial spokes (RSs), outer and IDAs, the CSC, and the N-DRC (Fig. 2). The outer dynein arms repeat once every $24 \mathrm{~nm}$ (Fig. 2) and are relatively homogeneous in structure (King and Kamiya 2009; King 2012; Kikkawa 2013; Kamiya and Yagi 2014; Owa et al. 2014). The subunit composition of the outer dynein arms in Chlamydomonas and humans is listed in Table 1 (Hom et al. 2011). The outer dynein arms are responsible for control of beat frequency and provide much of the power required for movement (Gibbons and Gibbons 1973; Kamiya and Okamoto 1985; Mitchell and Rosenbaum 1985; King and Kamiya 2009; Kamiya and Yagi 2014). Phenotypic analyses of outer dynein arm mutants have revealed that the dynein heavy chain (DHC) subunits 


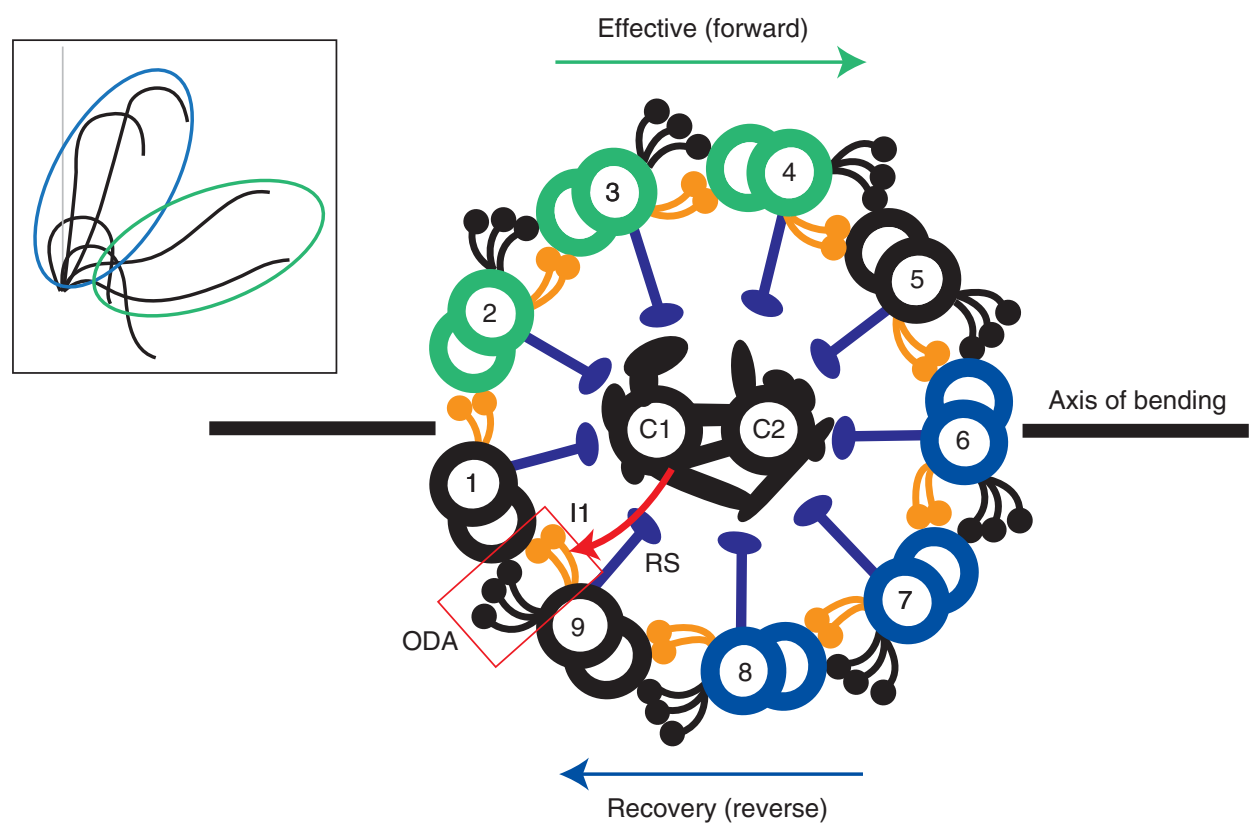

\begin{abstract}
Figure 1. Cross section of the axoneme and illustration of the "switching model" for alternating forward and reverse bends. The $9+2$ structure comprises nine outer doublet microtubules and a pair of singlet microtubules, $\mathrm{C} 1$ and $\mathrm{C} 2$, collectively known as the central pair. The outer doublet anchors the outer dynein arms (ODA), the radial spokes (RS), and the inner dynein arms, including I1 dynein. The black line passing through doublet \#6 and between doublets \#1 and \#2 represents the plane/axis of bending. According to the switching model, when dyneins on one side of the axis are active (i.e., on doublets $\# 2$, \#3, and \#4), microtubule sliding can result in the forward, or effective, bend (green in inset). When the direction of bending reverses, the dyneins on doublets \#2, \#3, and \#4 are inactivated, and dyneins on doublets $\# 6, \# 7$, and $\# 8$ are switched on to generate the recovery, or reverse, bend (blue in inset). (Adapted, with permission, from Smith 2007, (C) Journal of Cell Biology.)
\end{abstract}

$\alpha, \beta$, and $\gamma$ play distinct roles in regulation of flagellar motility. For example, the $\gamma$ DHC interacts with the light chains LC1 and LC4 (King and Patel-King 2012; Ichikawa et al. 2014). The $\gamma \mathrm{DHC}-\mathrm{LC} 1-\mathrm{LC} 4$ complex is thought to be important for part of the $\mathrm{Ca}^{2+}$ response and for feedback control in response to microtubule curvature (Morita and Shingyoji 2004; Hayashi and Shingyoji 2008; King 2010; King 2013). Additional models for the roles of the outer and IDAs and other functional domains in the outer dynein arms have been reviewed elsewhere (King and Kamiya 2009; Kamiya and Yagi 2014).

Based on analysis of wild-type and mutant Chlamydomonas cells, the IDAs are much more complex and contain at least 12 different DHC species (Porter et al. 1996; Yagi et al. 2009). The DHCs are organized into seven different major dyneins (labeled $a, b, c, d, e, f / \mathrm{I} 1$, and $g$ ) and at least three minor dyneins (DHC3, DHC4, and DHC11) that are distinct in composition (Table 2) and location in the 96-nm repeat (Fig. 2) (King and Kamiya 2009; Yagi et al. 2009; Bui et al. 2012; Heuser et al. 2012a; Kamiya and Yagi 2014). The IDAs are responsible for control of the size and shape of the forward and reverse ciliary bends.

\subsection{A Sliding Microtubule/Switching Model for Ciliary Bending}

Both structural and functional evidence supports a sliding microtubule model of ciliary bending. This evidence includes EM of cilia in defined bend positions (Satir 1968), observations by dark-field microscopy of dynein-driven microtubule sliding in protease-treated axonemes (Summers and Gibbons 1971), and direct demonstration that microtubule sliding occurs during axonemal bending (Shingyoji et al. 1977; Brokaw 1989).

Diverse data also support a switching model for alternating the pattern of forward and reverse bends (Satir and Matsuoka 1989; Smith 2007). The basis for this model stems from the discovery that axonemal dyneins are minus end-directed motors - that is, when active, dynein and its associated A tubule move in the proximal direction relative to the B tubule of the adjacent doublet (Sale and Satir 1977). Therefore, for ciliary bending to occur, the dyneins on all doublets cannot be active at the same time. Rather, there must be a switching mechanism that controls the activity of the dynein motors on different doublets and 


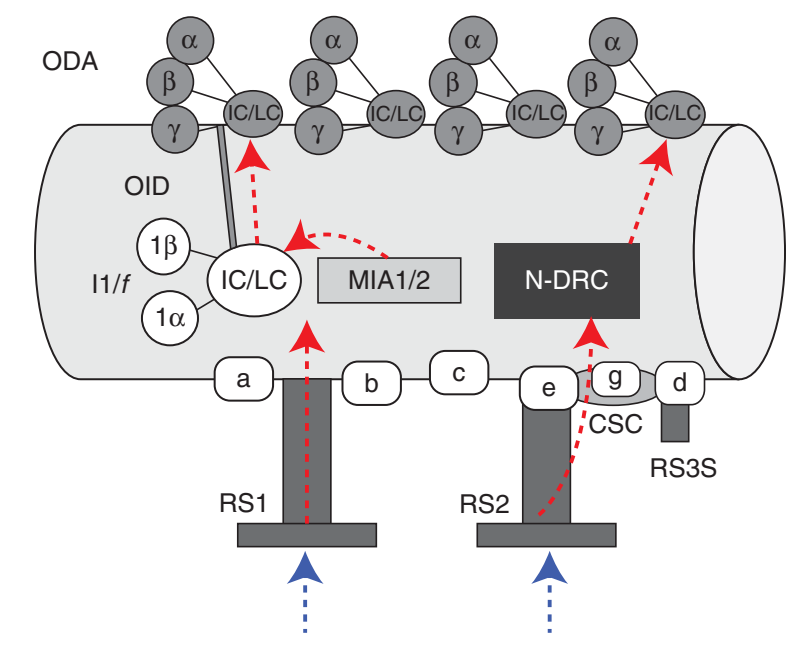

Figure 2. Parallel control pathways associated with the two regulatory hubs present in the 96-nm repeat: (1) RS1 and I1 dynein-MIA complex, and (2) RS2, CSC, and N-DRC. The blue arrows indicate chemical and mechanical signals that emanate from the central pair (CP) projections and are transmitted through the radial spoke heads to the bases of RS1 and RS2. The red arrows indicate signals transmitted from RS1 to the I1 dynein-MIA complex and from RS2 to the CSC and N-DRC, respectively, to control ciliary motility. The outer dynein arms (ODA) are also connected to both the I1-MIA complex and the N-DRC, also shown by the red arrows. CSC, calmodulin- and spoke-associated complex; N-DRC, nexin-dynein regulatory complex; IC, intermediate chain; LC, light chain; MIA, modifier of inner arms; RS, radial spoke. (Adapted from Yamamoto et al. 2013; King 2013.)

on opposite sides of the axis of bending (Fig. 1). Thus, the dyneins on one side of the axis of the axoneme are responsible for generating the forward bend, and the dyneins on the other side of the axis of the axoneme must produce the reverse bend. The most dramatic evidence for switching in the axoneme comes from the Shingyoji laboratory in which the investigators were able to activate dynein-driven sliding by physically bending doublet microtubules in isolated axonemes (Morita and Shingyoji 2004). Shingyoji and collaborators further determined that bending the doublet microtubules in the opposite direction activated microtubule sliding in the reverse direction (Hayashi and Shingyoji 2008). These results are consistent with regulation of dyneins by bending or structural distortion of the axoneme (Brokaw 1985; Lindemann and Mitchell 2007) and a switching model for alternating bend directions.

Further tests of the models will require a clear definition of active and nonactive states of the dynein motors. In the original studies by Goodenough and Heuser, the outer arms were observed in two different conformations, depending on the presence or absence of ATP (Goodenough and Heuser 1982). Recent cryo-ETanalysis has also revealed distinct structures for the outer dynein arms in the presence or absence of ADP-vanadate (Movassagh et al. 2010). In addition, cryo-ET of live sea urchin sperm flagella captured during movement by rapid freezing has revealed distinct structural conformations of the outer dynein arms on different outer doublet microtubules (see Supplemental Fig. 1 in Lin et al. 2014a).

Other key regulatory components of the 96-nm repeat include the two (or three) RSs and their associated substructures. Each RS is part of a distinct signaling hub: (a) RS1 is associated with IDAs 1 (I1 dynein or dynein $f$ ) and the MIA (modifier of inner arms) complex (King and Dutcher 1997; Wirschell et al. 2007; Yamamoto et al. 2013; Lin et al. 2014b), and (b) RS2 is associated with the CSC and N-DRC (Dymek et al. 2011; Heuser et al. 2012b; Porter 2012). Each hub is discussed in detail in Sections 3 and 4 below.

\subsection{RS Heterogeneity}

Common to both regulatory hubs are the RSs that are crucial for the regulation of dynein-driven motility as mutations that disrupt the assembly of the RSs typically result in complete flagellar paralysis (Witman et al. 1978). In vitro assays using isolated axonemes from wild-type and RS mutants further showed that the RSs increase microtubule sliding velocities, presumably through control of the dyneins (Smith and Sale 1992a). In most species, the RSs repeat as a set of triplets (RS1, RS2, RS3) every $96 \mathrm{~nm}$ along the length of the A tubule (Lin et al. 2014b). However, in a few organisms such as Chlamydomonas, the RSs repeat as pairs, RS1 and RS2, and a smaller structure, the RS stump or stand-in (RS3S), marks the position normally occupied by RS3 (Fig. 2) (Pigino et al. 2011; Barber et al. 2012; Lin et al. 2012). Biochemical studies have revealed that the RSs are large complexes comprising at least 23 different subunits (RSPs) that include a diverse group of structural and signaling proteins (Table 3) (Yang et al. 2006). These subunits have been assigned to specific regions of the RSs (e.g., head, neck, stalk, and base) by the analysis of different mutants and cryo-ET (Oda et al. 2014b).

In all species examined thus far, the detailed substructures of RS1 and RS2 are very similar, but RS2 contains two additional densities at its base, where it attaches to the A tubule (Pigino et al. 2012). The specific proteins located in the two additional RS2 densities are still unknown, but they probably correspond to a small subset of the RSPs identified previously as components of the RS stalk in Chlamydomonas (see Table 3) (Yang et al. 2006). Given that this region is the site of contact between RS2 and both the CSC and the N-DRC, it is also likely that the unique proteins in the additional RS2 densities, such as FAP206 (Vasudevan et al. 2015), play a role in mediating interactions among the three complexes (see Sec. 3). 


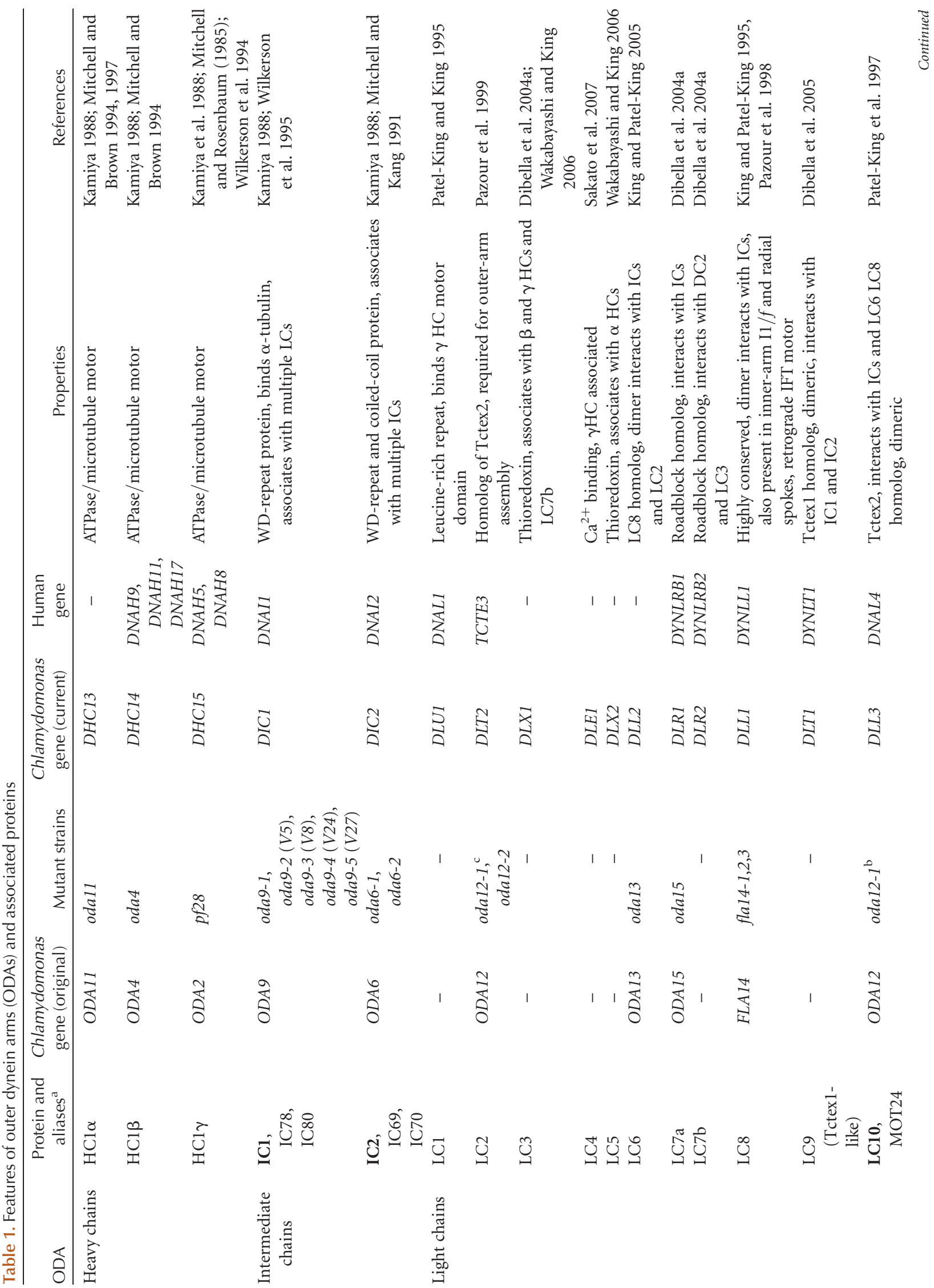


Downloaded from http://cshperspectives.cshlp.org/ on April 26, 2023 - Published by Cold Spring Harbor Laboratory Press

R. Viswanadha et al.
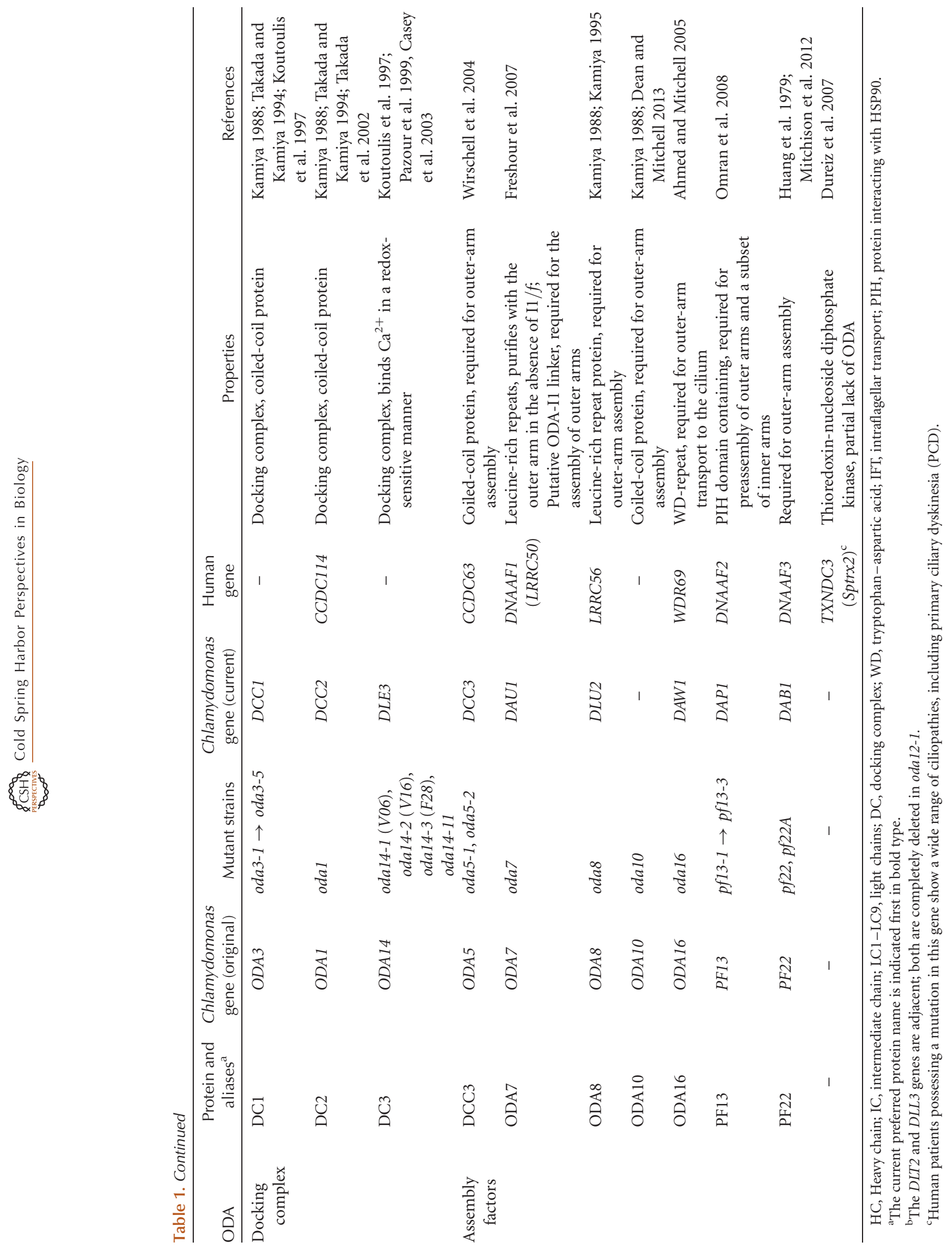


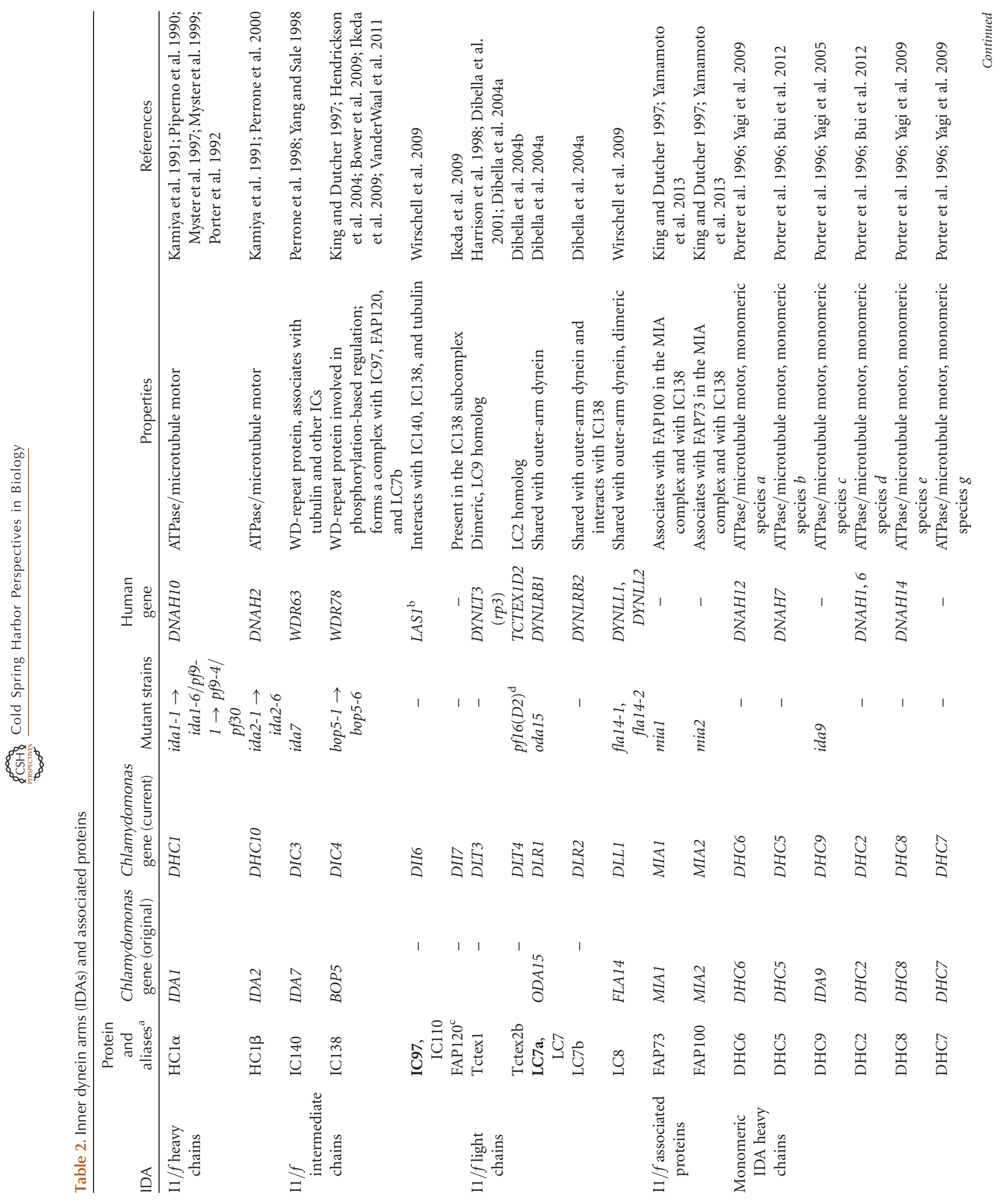




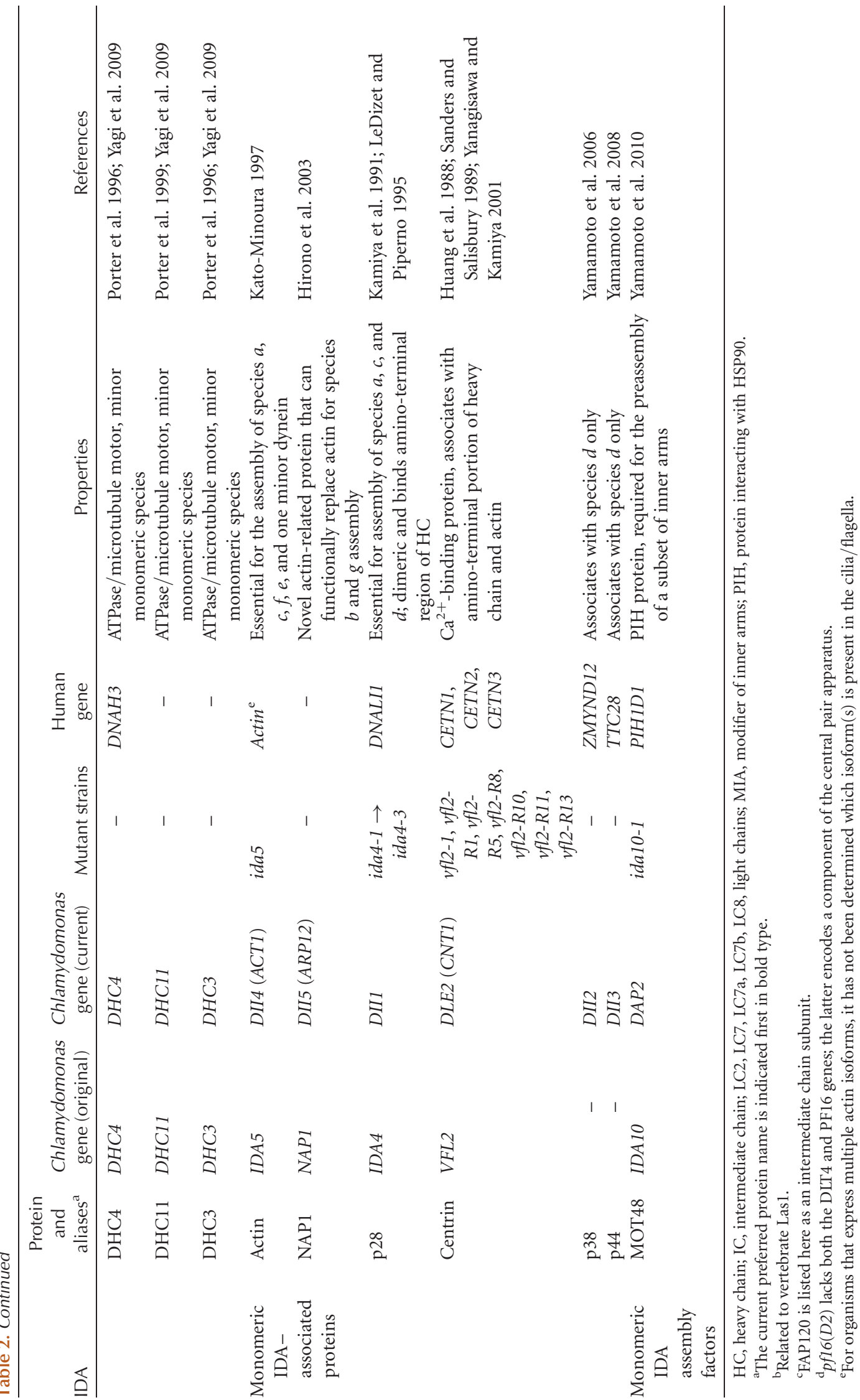


Table 3. Radial spoke-associated proteins (RSPs) and calmodulin- and spoke-associated complex proteins (CaM-IPs)

\begin{tabular}{|c|c|c|c|c|c|}
\hline Chlamydomonas name & MW (kDa) & Motifs & Position & Homo sapiens name & References \\
\hline RSP1 & 78.6 & MORN & Head & RSPH1 & Yang et al. 2006 \\
\hline RSP2 & 77.4 & DPY, GAF, CaM binding & Neck & DYDC2 & Yang et al. 2006 \\
\hline RSP3 & 56.8 & AKAP motif & Stalk & RSPH3 & Williams et al. 1989 \\
\hline RSP4 & 49.8 & Unknown & Head & RSPH4A/6A & Curry et al. 1992 \\
\hline RSP5 & 48.8 & Aldo-keto reductase & Stalk & - & Yang et al. 2006 \\
\hline RSP6 & 48.8 & Unknown & Head & RSPH4A/6A & Curry et al. 1992 \\
\hline RSP7 & 55 & RII a/EF hand & Stalk & CALML5 & Yang et al. 2006 \\
\hline RSP8 & 40.5 & Armadillo & Stalk & ARMC4 & Yang et al. 2006 \\
\hline RSP9 & 29.5 & Unknown & Head & RSPH9 & Yang et al. 2006 \\
\hline RSP10 & 23.5 & MORN & Head & RSPH1/10B & Yang et al. 2006 \\
\hline RSP11 & 21.5 & RII a & Stalk & ROPN1L & Yang et al. 2006 \\
\hline RSP12 & 19.7 & Peptidyl-prolyl isomerase & Stalk & PPIL6 & Yang et al. 2006 \\
\hline RSP13 & $\sim 98$ & Unknown & Stalk & - & Yang et al. 2006 \\
\hline RSP14 & 28.3 & Armadillo & Stalk & RTDR1 & Yang et al. 2006 \\
\hline RSP15 & $\sim 38$ & LRR & Stalk & - & Yang et al. 2006 \\
\hline RSP16 & 39 & DnaJ/DnaJ-chaperone & Neck & DNAJB13 & Yang et al. 2006 \\
\hline RSP17 & 98.5 & GAF & Stalk & - & Yang et al. 2006 \\
\hline RSP18/CaM-IP2 ${ }^{\mathrm{a}}$ & 183 & AKAP/AAT-1, IQ & Stalk/CSC & C3orf15, MAATS1 & Dymek and Smith 2007 \\
\hline RSP19/CaM-IP3 ${ }^{\mathrm{a}}$ & 140 & Pyridine-disulfide oxidoreductase & Stalk/CSC & C20orf26 & Dymek and Smith 2007 \\
\hline RSP20 & 18.3 & EF hand (calmodulin) & Stalk/CSC & Calmodulin & Yang et al. 2001 \\
\hline RSP21 & 16 & - & Stalk & - & Yang et al. 2006 \\
\hline RSP22/LC8 & 10.3 & LC8 & Stalk & DYNLL2 & Yang et al. 2009 \\
\hline RSP23 & 61 & NDK, DPY, IQ & Neck & NME5 & Patel-King et al. 2004 \\
\hline CaM-IP4 ${ }^{\mathrm{a}}$ & 100 & WD repeat & Stalk/CSC & WDR66 & Dymek and Smith 2007 \\
\hline
\end{tabular}

AKAP, A kinase anchoring protein motif; LRR, leucine-rich repeat; MORN, membrane occupation and recognition nexus; NDK, nucleoside diphosphate kinase motif; WD, WD40 repeat; DPY, Dpy-30 motif; GAF, cGMP, adenylyl cyclase, FhIA domain; IQ, calmodulin-binding domain; CSC, calmodulin- and spokeassociated complex.

${ }^{\mathrm{a} C a M}-\mathrm{IP} 2$, CaM-IP3, and CaM-IP4 are also known as FAP91, FAP61, and FAP251 (Dymek and Smith 2007).

\section{REGULATION OF AXONEMAL BENDING BY I1/ $f$ DYNEIN AND THE MIA COMPLEX LOCATED NEAR RS1}

\subsection{I1/ $f$ Dynein Is a Conserved Two-Headed Inner Dynein Arm}

The number and complexity of inner-arm dyneins have made it challenging to define their individual contributions to motility, but nearly all the inner-arm mutants characterized thus far display some defects in their flagellar waveform (Kamiya and Yagi 2014). In particular, several studies have shown that I1 $f$ dynein is required for normal control of ciliary bending (Brokaw and Kamiya 1987; Bayly et al. 2010; VanderWaal et al. 2011). For example, Chlamydomonas mutants that fail to assemble I1 dynein show slow, smooth forward swimming, decreased dynein-driven microtubule sliding, abnormal ciliary waveforms and reduced phototaxis (reviewed in Wirschell et al. 2007; see also Porter and Sale 2000; Wirschell et al. 2007; Kamiya and Yagi 2014). However, the precise mechanism by which I1 dynein modulates ciliary bending remains unknown.

I1 dynein is the only inner-arm subspecies that contains two distinct DHCs. Each I1 DHC is highly conserved and widely distributed among ciliated eukaryotes (Wickstead and Gull 2007). Additional unique features of I1 dynein suggest that I1 dynein and the MIA complex (King and Dutcher 1997; Yamamoto et al. 2013) play an important role in control of ciliary bending. We discuss models in which I1 dynein could operate through (a) a direct interaction with other axonemal dyneins to regulate their motor activity and (b) resisting microtubule sliding driven by other axonemal dyneins.

I1 dynein was first identified by analysis of slow swimming mutants (Piperno et al. 1990; Kamiya et al. 1991; Porter et al. 1992). It is located in the proximal end of each 96-nm repeat near RS1 (Figs. 1 and 2), and it makes contact with many other structures in the axoneme, including the ODA and neighboring single-headed IDAs (Heuser et al. 2012a). I1 dynein can be easily extracted from isolated axonemes and purified as an intact $20 S$ complex (Piperno et al. 1990; Porter et al. 1992) comprising two heavy chains ( $1 \alpha$ and $1 \beta$ ), three intermediate chains (IC97, IC140, and IC138), and five light chains (Tctex1, Tctex2b, LC7a, LC7b, and LC8) (Fig. 3 inset; Tables 1 and 2). In addition, partial complexes of I1 dynein can assemble in the absence of IC138 and associated proteins defining a "IC138 sub- 
R. Viswanadha et al.

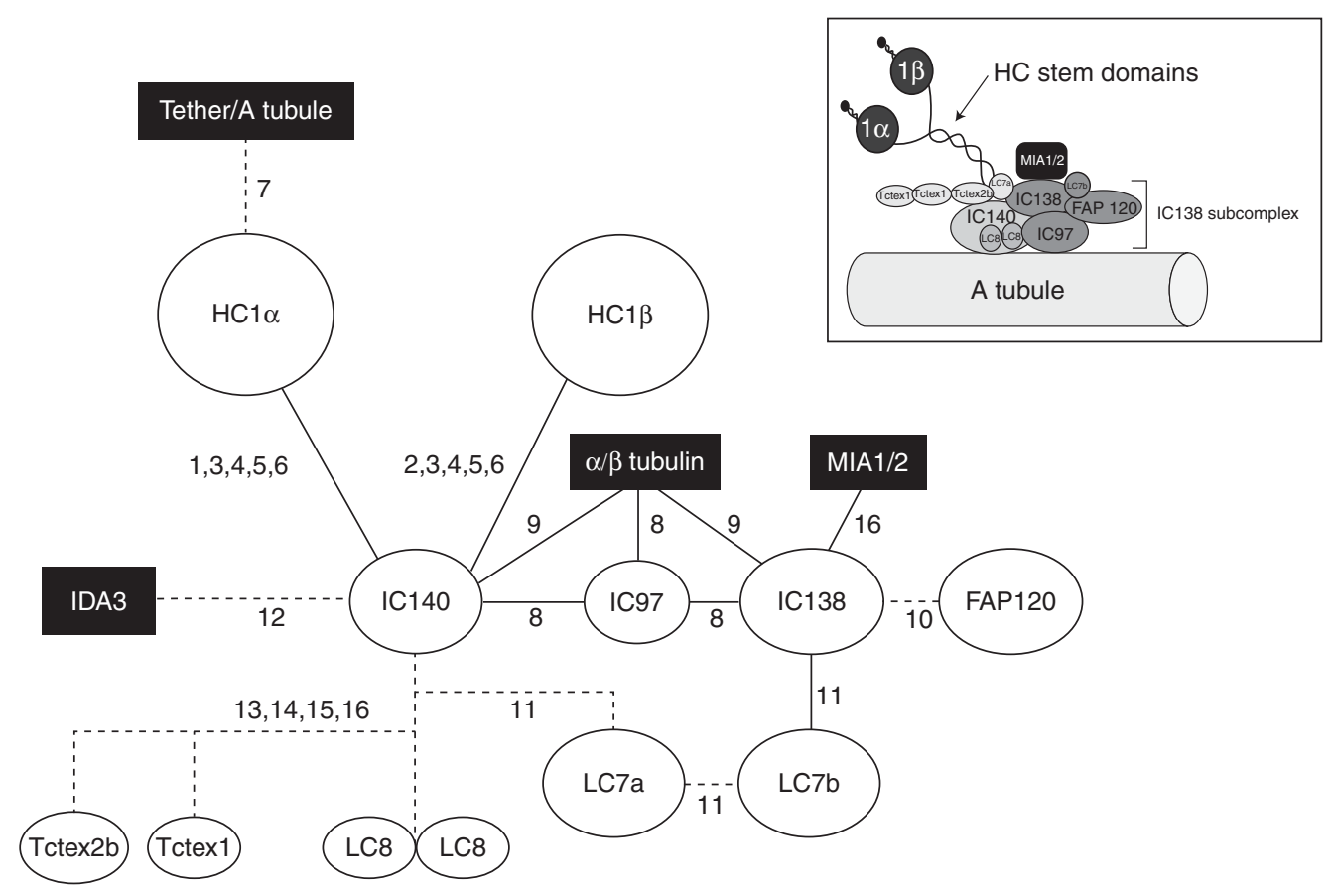

Figure 3. Diagram of interactions within I1 dynein and with the MIA complex and associated proteins. As illustrated in the inset, I1 dynein contains two distinct heavy chain motor domains ( $\mathrm{HCl} \alpha$ and $\mathrm{HC} 1 \beta)$, three intermediate chains (IC140, IC97, and IC138), and five light chains (Tctex1, Tctex2b, LC8, LC7a, and LC7b). The solid lines represent protein-protein interactions determined by biochemical and/or structural analysis of I1 dynein assembly mutants (1: Myster et al. 1997; 2: Perrone et al. 2000; 3: Bower et al. 2009; 4: Toba et al. 2011; 5: VanderWaal et al. 2011; 6: Perrone et al. 1998; 8: Wirschell et al. 2009; 9: Hendrickson et al. 2004; 11: DiBella et al. 2004a; 16: Yamamoto et al. 2013). The dashed lines indicate predicted interactions based on the analysis of I1 assembly mutants (6: Perrone et al. 1998; 7: Heuser et al. 2012a; 10: Ikeda et al. 2009; 11: DiBella et al. 2004a; 12: Viswanadha et al. 2014; 13: Wu et al. 2005; 14: Harrison et al. 1998; 15: Yang et al. 2009; 16: DiBella et al. 2004b). (Adapted from DiBella et al. 2004a; King 2013.)

complex" comprising IC138, IC97, FAP120, and LC7b (Fig. 2, inset) (Hendrickson et al. 2004; Bower et al. 2009; Wirschell et al. 2009). Analysis of IC138 (bop5) mutants has revealed that the IC138 subcomplex, like I1 dynein, is essential for control of ciliary waveform (Brokaw and Kamiya 1987; Bayly et al. 2010; VanderWaal et al. 2011).

\subsection{The MIA Complex Interacts with I1 Dynein}

I1 dynein has been shown to physically interact with the MIA complex that was discovered by genetic, biochemical, and structural analysis of Chlamydomonas mutants defective in light-regulated movement (phototaxis) (King and Dutcher 1997; Yamamoto et al. 2013). Cryo-ET analysis of wild-type and mia mutants revealed that the MIA complex is located near the IC138 complex between I1 and the NDRC (Fig. 2) (Yamamoto et al. 2013). The complex comprises two coiled-coil protein subunits MIA1 and MIA2. Motility analysis of the mutants mia1 and mia2 revealed a slow swimming phenotype similar to that of mutants lacking I1 dynein. Further genetic and phenotypic analysis revealed that I1 dynein and the MIA complex might act as a functional unit to control ciliary movement (Yamamoto et al. 2013).

\subsection{Mutations Reveal Functional Domains in I1 Dynein}

Several mutations in I1 subunits have been extremely useful in providing detailed information on the roles of individual subunits in the assembly of the I1 complex and/or function of the I1 dynein complex in ciliary motility (Bower et al. 2009; Wirschell et al. 2009; Toba et al. 2011; VanderWaal et al. 2011; Heuser et al. 2012a). For instance, most mutations in genes that encode $1 \alpha$ and $1 \beta$ DHCs or the intermediate chain subunit IC140 result in a complete failure in I1 dynein assembly, leaving a gap in every $96-\mathrm{nm}$ repeat (Piperno et al. 1990; Kamiya et al. 1991; Porter et al. 1992; Myster et al. 1997; Perrone et al. 1998; Myster et al. 1999; Perrone et al. 2000). Notably, loss of the I1 dynein does not affect the assembly of other IDAs, RSs, outer-arm dyneins, or the MIA complex, indicating that the targeting and assembly of I1 dynein is independent of other axonemal components (Piperno et al. 1990; Kamiya et al. 1991; Porter 
et al. 1992; Smith and Sale 1992b; Bui et al. 2012; Heuser et al. 2012a; Yamamoto et al. 2013). Thus, interpretation of I1 mutant phenotypes (Brokaw and Kamiya 1987; Bayly et al. 2010; VanderWaal et al. 2011) is not complicated by failure of assembly of additional axonemal components.

Interestingly, however, both I1 DHC mutations can be partially rescued by transformation with DHC constructs lacking the dynein motor domains, indicating that the motor domains are not required for the assembly of the I1 complex (Myster et al. 1999; Perrone et al. 2000). The mutant strains lacking either the $1 \alpha$ or $1 \beta$ motor domains assemble the remainder of I1 dynein at the correct location in the axoneme (Myster et al. 1999; Perrone et al. 2000). These studies pinpointed the location of both dynein-motor domains within the structure of the 96-nm repeat (Fig. 2). Toba and colleagues (Toba et al. 2011) took advantage of these mutants to show that each motor domain has distinct properties in in vitro microtubule sliding and translocation assays. The investigators proposed that one of the functions of I1 dynein is inhibition of microtubule sliding driven by other axonemal dyneins (Toba et al. 2011). Consistent with this model, Kotani and colleagues (Kotani et al. 2007) used in vitro microtubule translocation experiments to show that purified I1 dynein, when mixed with purified IDA $c$, impeded microtubule translocation driven by dynein $c$.

These data support a model in which I1 dynein acts as a brake to slow and locally regulate sliding driven by the outer dynein arm and other IDAs. Consistent with this model, studies of the double mutants lacking I1 dynein and the RSs or the central pair (both of which are required for dynein-driven sliding), display an increase in microtubule sliding velocities compared with RS or central pair mutants alone (Yang and Sale 2000; Smith 2002). One interpretation of this observation is that the increase in velocity of sliding in mutants lacking I1 dynein is due to the loss of resistance to microtubule sliding.

Thus, the I1 dynein appears to play two distinct rolescontributing to microtubule sliding on the one hand, but also resisting microtubule sliding by other dyneins. CryoET later revealed that the two I1 motor domains differ with respect to their connections to other structures within the axoneme. The $1 \beta$ motor domain is closer to the IC-LC complex and the outer dynein arms, whereas the $1 \alpha$ motor domain is closer to its neighboring inner-arm dynein and is also tethered to the underlying A tubule (Heuser et al. 2012a). These observations are consistent with a model in which the $1 \beta$ dynein is the primary contributor to force production, and the $1 \alpha$ dynein is the motor domain that resists or limits microtubule sliding.

In another instance, mutations in the BOP5 locus have defined an IC138 regulatory subcomplex within the I1 dynein (Fig. 3, inset) (Bower et al. 2009; Wirschell et al. 2009;
Heuser et al. 2012a). This subcomplex is also important for control of microtubule sliding, bend propagation, and the axonemal waveform (Brokaw and Kamiya 1987; Bayly et al. 2010; VanderWaal et al. 2011). Comparative analysis of the I1 structure between wild-type and bop 5 mutant axonemes by thin-section EM and cryo-ET confirmed the location of the IC138 complex at the proximal end of the 96-nm repeat, just above RS1 (Bower et al. 2009; Heuser et al. 2012a). In addition, as expected for a regulator of motility, cryo-ET analysis also revealed that the IC-LC complex of the I1 dynein interacts with multiple structures in the axoneme (Heuser et al. 2012a). These interactions include a link to the outer dynein arms, a link to inner-arm $a$ (IA2), and a link to the MIA complex (Heuser et al. 2012a; Yamamoto et al. 2013). This IC-LC complex is thought to mediate signals to the two different I1 dynein motor domains, based in part on the phosphorylation state of the IC138 subunit.

\subsection{Regulation of I1 Dynein by Axonemal Kinases and Phosphatases and IC138 Phosphorylation}

In addition to its numerous structural interactions with other axonemal components (Heuser et al. 2012a), the activity of I1 dynein in the axoneme appears to be regulated by changes in phosphorylation of the intermediate chain subunit IC138 (Habermacher and Sale 1997; King and Dutcher 1997; Yang and Sale 2000; Bower et al. 2009; Wirschell et al. 2009). Although the mechanism of regulation by IC138 is not understood, the protein kinases (Yang and Sale 2000; Gaillard et al. 2001; Gokhale et al. 2009) and phosphatases thought to control IC138 phosphorylation (Elam et al. 2011) are physically anchored in the axoneme and are presumably in close proximity to IC138. The data support a model in which IC138 is the regulatory phosphoprotein that controls I1 dynein activity.

As mentioned above, the MIA complex contains two coiled-coiled proteins, FAP73 (MIA1) and FAP100 (MIA2), and both mutants show an altered waveform, slightly reduced beat frequency, and defective phototaxis-all of which are characteristic of defects in assembly and/or function of I1 dynein. Because the IC138 subunit was hyperphosphorylated in the mia mutants, the investigators postulated that the MIA complex exerts its regulatory effects through the kinases and phosphatases responsible for IC138 phosphorylation (Yamamoto et al. 2013). One model is that the MIA complex functions by correctly positioning the enzymes relative to the IC138 substrate to ultimately control ciliary waveform (King 2013; Yamamoto et al. 2013). In addition, the mia mutants show a slightly reduced beat frequency, suggesting a functional interaction between I1 and the outer arms (Yamamoto et al. 2013). Consistent with these observations, 
cryo-ET has revealed a physical link between the IC-LC complex of I1 dynein and the outer dynein arm (Nicastro et al. 2006; Bui et al. 2009; Bui et al. 2012; Heuser et al. 2012a; Oda et al. 2013).

\subsection{A Model for Local Control of Microtubule Sliding and Regulation of Ciliary Bending by I1 Dynein}

One of the current questions concerns how the central pair/RS structures regulate the activity of I1 dynein. Structural evidence indicates that RSs can, in part, operate as mechanical transducers that can limit microtubule sliding (Warner and Satir 1974). Recent studies by Oda and colleagues (Oda et al. 2014b) indicate that a physical interaction between the spoke heads and central pair projections is required for regulating microtubule sliding and motility. One idea, illustrated in Figure 1, is that in wild-type axonemes, I1 activity is locally regulated by signals emanating from the central pair and directed to specific outer-doublet microtubules (Wargo and Smith 2003; Mitchell and Nakatsugawa 2004). In this model, signals, both chemical and mechanical in nature, from the asymmetric central pair are transmitted through the RSs to a specific subset of doublets, presumably on one side of the axis of the axoneme, to locally regulate I1 dynein activity (Fig. 1, curved red arrow). I1 dynein might act directly to regulate other dyneins or as a brake to impede sliding. Either way, the form of the bend would be altered, resulting in changes in the shape of the forward or the reverse bend or both (Porter and Sale 2000; Wirschell et al. 2007). Based on this general model and other features discussed in this section, a number of questions arise: What are the signals leading from the central pair to the outer doublets? How are signals from the central pair directed to specific outer doublets? How are signals from RS1 transmitted to the I1 dynein? Does the I1 dynein-MIA complex regulate the activity of other dyneins directly and/or operate as a "brake" to resist microtubule sliding?

\section{THE RS2 REGULATORY COMPLEX AND DOWNSTREAM REGULATION}

\subsection{The Calmodulin- and Spoke-Associated Complex}

The CSC was first identified as a group of three polypeptides that coimmunoprecipitated with calmodulin (CaM) and RS subunits in extracts prepared from wild-type axonemes (Dymek and Smith 2007). The calmodulin- and spoke-associated complex proteins (CaM-IP) polypeptides also cosedimented with the RS subunits at $\sim 20 S$ on sucrose density gradients. However, when extracts were prepared from mutants lacking RS heads, the CSC subunits cosedi- mented with the RS stalks at $\sim 17 S$. Furthermore, when extracts from mutants lacking the RS were analyzed, the CSC subunits formed a smaller complex at $\sim 11 S$. Blot overlay assays showed that one CSC subunit, CaM-IP2, binds directly to RSP3, an A-kinase anchoring protein (AKAP) located at the base of the RS stalk (Gaillard et al. 2001; Sivadas et al. 2012). Collectively, these data suggested that the CSC is tightly associated with the base of the RS stalk, but part of a distinct complex. However, it remained uncertain whether the CSC is associated with RS1 or RS2. A possible association with RS1 was suggested by the observation that treatment of central pair or RS mutant axonemes with an antibody against CaM-IP2 could increase microtubule sliding velocities, but only if the I1 dynein was also present (Dymek and Smith 2007). However, the coimmunoprecipitation of the CSC subunits with CaM was disrupted in the $d r c$ mutants $p f 2$ and $p f 3$, suggesting a possible association with RS2. Resolution of this question would require the isolation of mutants that lack most of the CSC subunits.

The development of artificial microRNA (amiRNA) methods to knockdown expression of CaM-IP2 and -IP3 has resulted in the isolation of several strains that assemble reduced amounts of all three CSC subunits in isolated axonemes (Dymek et al. 2011). Biochemical comparisons further showed that CaM-IP2 and -IP3 correspond to RSP18 and -19, respectively, two RS subunits present in lower abundance than other RSPs (Yang et al. 2006; Heuser et al. 2012b). CSC amiRNA mutants swim forward with reduced swimming velocities owing to their shallow waveforms and a lack of coordination between their two flagella (Dymek et al. 2011). Assembly of the RS is variable and reduced when viewed by thin-section EM. Analysis by cryoET revealed that $\sim 50 \%$ of the $96-\mathrm{nm}$ repeats contain RS pairs, $\sim 25 \%$ of the repeats lack RS2, and the remaining 96$\mathrm{nm}$ repeats contain either an additional spoke or are too ambiguous to score. These observations strongly suggested that the CSC is associated with RS2 (Dymek et al. 2011). Subtomogram averaging further showed that the CSC mutants display variable defects in the structures of four other axonemal complexes in addition to RS2 (Fig. 4) (Heuser et al. 2012b). These include (1) the homolog of the third RS, known as RS3S; (2) some of the IDAs (IDA $e$, IA4; IDA $a$, IA2; and IDA $d$, IA6); (3) two regions of the N-DRC, one connecting to the tail of IDA $e$ (IA4) and another connecting the N-DRC baseplate to RS2; and (4) a hole in the Btubule inner junction near the base of RS2 and the N-DRC (Heuser et al. 2012b). Given the heterogeneity in RS assembly, the subtomogram averages were sorted into two classes, the first containing RS2 and the second lacking RS2. The class averages made it possible to identify those structural defects most closely correlated with the loss of CSC sub- 


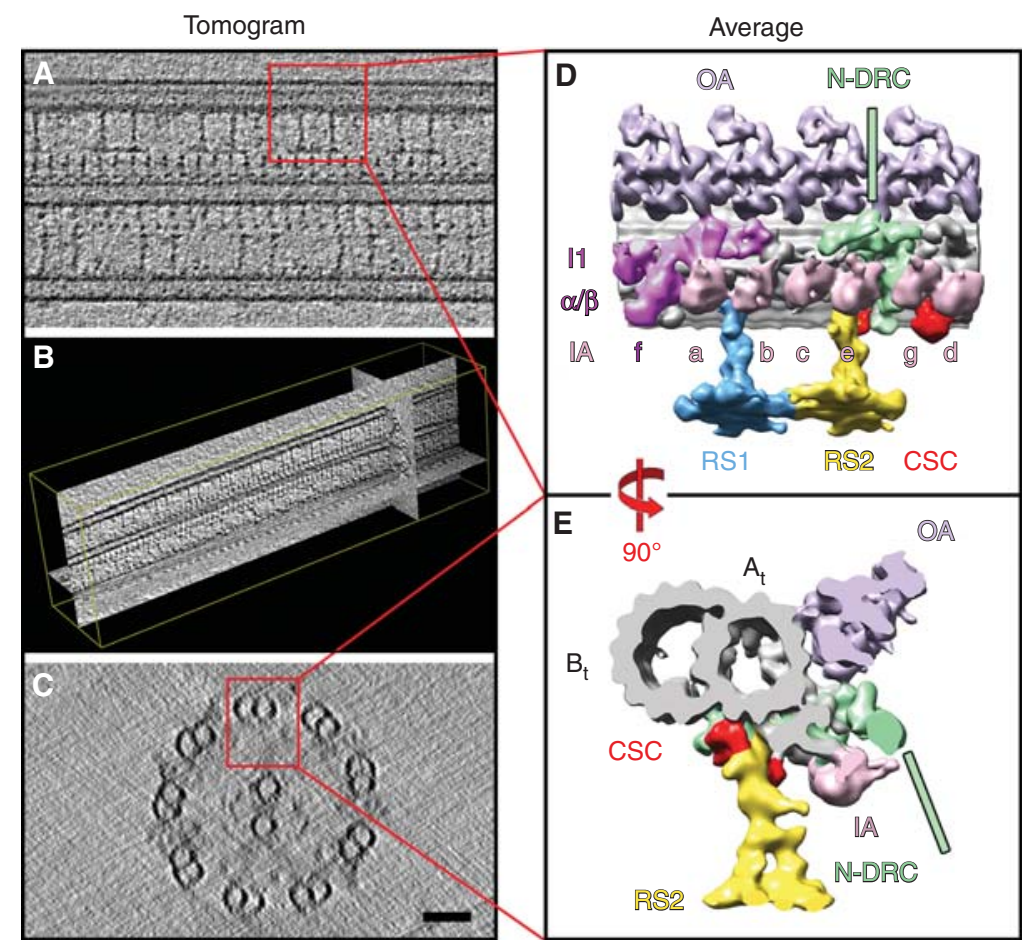

Figure 4. The Chlamydomonas axoneme ultrastructure. $(A-C)$. Cryo-electron tomography slices show $(A)$ a longitudinal view, $(B)$ a 3D view, and $(C)$ a cross section of a Chlamydomonas axoneme. The red boxes highlight one 96$\mathrm{nm}$ axonemal repeat unit in both views. $(D, E)$ Isosurface renderings show an averaged 96-nm axonemal repeat in $(D)$ longitudinal and $(E)$ cross-sectional orientation. The cross-sectional slice is taken close to radial spoke 2, viewing from the proximal to the distal end. Key axonemal structures are highlighted: A- and B-tubule $\left(A_{t}, B_{t}\right)$, nexin-dynein regulatory complex (N-DRC), radial spokes (RS1,RS2), calmodulin- and spoke-associated complex (CSC), and inner and outer dynein arms (IA,OA). Inner-arm dyneins include the I1 complex (dynein $f \alpha$ and $\beta$ heavy chain motor domains) and dyneins a-g. (Adapted from Satir et al. 2014; originally from Heuser et al. 2012b, with permission of the American Society for Cell Biology; permission conveyed through Copyright Clearance Center, Inc.; Heuser et al. 2012a.)

units. Comparisons with other RS and N-DRC mutants have led to the following model shown in Figure 4: the CSC subunits are located close to or within the two unique RS2 densities (the back prong of the RS2 base and the RS2to-N-DRC connector), as well as part of RS3S (Heuser et al. 2012b). In addition, the CSC interacts with the baseplate of the N-DRC and physically interconnects the base of RS2 to the base of RS3S (see also Fig. 6 of Heuser et al. 2012b). How the individual CSC subunits might be arranged within these different structures is still unknown. Furthermore, there might be additional, as yet unidentified, subunits of the RS, N-DRC, and/or CSC that contribute to the assembly of this highly interconnected, regulatory hub.

\subsection{Genetic Analyses of the Dynein Regulatory Complex}

Early efforts to identify other components involved in signaling between the RS and the dynein arms were based on the observation that most RS and CP mutations resulted in flagellar paralysis. The isolation of extragenic suppressors that could restore some motility to RS/CP mutants resulted in the identification of five loci, known as SUP-PF1SUP-PF5 (Huang et al. 1982; Piperno et al. 1994). Doublemutant analyses showed that two motility mutants, $p f 2$ and $p f 3$, could also suppress RS/CP paralysis (Huang et al. 1982). Molecular and biochemical studies sorted the seven loci into two groups, one group that alters the activity of the outer dynein arms and flagellar beat frequency, and a second group that appears to alter the activity of the IDAs and the flagellar waveform (Huang et al. 1982; Brokaw and Kamiya 1987; Piperno et al. 1992; Gardner et al. 1994). The first group is represented by the sup-pf1 and sup-pf2 alleles in the genes encoding the $\beta$ and $\gamma$ DHCs, respectively, of the outer dynein arm (Porter et al. 1994; Rupp et al. 1996). The sup-pf1 and sup-pf2 mutations specifically reduce outer dynein arm activity, but they do not block assembly of the outer dynein arm. These observations led to a model in which the $\mathrm{CP} / \mathrm{RS}$ are predicted to coordinate dynein activity by selectively stimulating the inner-arm dy- 
neins. In the absence of signals from the CP/RS, the IDAs are partially inhibited, leading to flagellar paralysis. However, motility can be restored either by mutations that stimulate inner-arm activity or by mutations that reduce outerarm activity.

The second group of mutations did not directly affect dynein arms, but were instead associated with defects in the assembly of a discrete subset of tightly bound axonemal proteins known as the dynein regulatory complex (DRC) (Huang et al. 1982; Piperno et al. 1992; Piperno et al. 1994). The precise location of the DRC subunits was initially unknown, but Brokaw and Kamiya (1987) noted that drc mutants displayed aberrant flagellar waveforms similar to those observed in inner-arm dynein mutants. Biochemical studies later confirmed that many $d r c$ mutants were also deficient in the assembly of a subset of inner-arm dyneins (Piperno et al. 1992; Gardner et al. 1994; Piperno et al. 1994). Comparison of wild-type and mutant axonemes by thin-section EM and computer-image averaging finally revealed that most $d r c$ mutants lacked a large, crescentshaped structure at the distal end of the $96-\mathrm{nm}$ repeat (Mastronarde et al. 1992; Gardner et al. 1994; Rupp and Porter 2003). Interestingly, this structure was located at the junction between the inner and outer dynein arms and RS2, ideally situated to mediate signals between these structures (Fig. 2). Mastronarde and colleagues noted that this location was close to the predicted attachment site of the nexin link, but the precise relationship between the DRC and the nexin link remained uncertain (Mastronarde et al. 1992).

\subsection{Identification of the DRC as the Nexin Link}

Early EM studies identified the nexin links as flexible filaments that interconnect the A tubule of one outer doublet microtubule to the B tubule of the adjacent outer doublet. They were first described in extracted Tetrahymena axonemes (Gibbons 1963) and then visualized in intact cilia following ninefold rotation of axoneme cross sections. Their presence in extracted ciliary remnants led to the proposal that the nexin links are crucial for maintaining the cylindrical structure of the nine doublet microtubules within the axoneme (Stephens 1970).

Analysis of longitudinal sections indicated that the nexin links repeat every $96 \mathrm{~nm}$ and are often inclined at variable angles (Warner 1976; Witman et al. 1978). Under experimental conditions that induced outer doublet microtubules to splay apart, the nexin links appeared to stretch (Warner 1976). However, other studies of actively bending axonemes suggested instead that the nexin links undergo cycles of lateral displacement along the B tubule (Warner 1983; Bozkurt and Woolley 1993; Woolley 1997).
The study of actively beating flagella by cryo-ET (Lin et al. 2014a) might soon resolve this apparent discrepancy.

The idea that the nexin links are the essential component that resists dynein-driven microtubule sliding was based on early studies with protease-treated axonemes. EM analysis of isolated axonemes indicated that protease treatment disrupted the nexin links and RSs with a timecourse that could be correlated closely with the extent of ATP-induced axoneme disintegration (Summers and Gibbons 1971). Furthermore, pf14 axonemes lacking RSs would not undergo microtubule sliding without protease treatment (Witman et al. 1978). These observations implicated the nexin links as the protease-sensitive structure resisting dynein activity.

Cryo-ET has resolved some of the mysteries associated with the nexin link. Images of wild-type axonemes showed that the nexin link is a very large structure $(\sim 1.5 \mathrm{MDa})$, with one end anchored to the A tubule, close to the base of RS2. The link wraps around the A tubule and then bifurcates as it reaches across the interdoublet space to make contact with the neighboring B tubule (Figs. 4 and 5) (Nicastro et al. 2006).

Later studies revealed that the various $d r c$ mutants lack different regions within the overall structure of the nexin link (Heuser et al. 2009). The missing regions define two major domains-the baseplate and the linker (Fig. 5). The baseplate is the region attached to the underside of the A tubule, extending from the A tubule-B tubule junction to protofilament A4 of the A tubule. This domain is $\sim 350 \mathrm{kDa}$, and it is significantly reduced in the $p f 3$ mutant. The baseplate also makes at least three connections to neighboring structures in the axoneme. These include (1) a thin connection between the end of the baseplate and the B11 protofilament adjacent to the A tubule-B tubule junction, (2) a connection between the baseplate and the tail of inner-arm dynein $e$ (IA4), and (3) a connection between the baseplate and RS2. As discussed above (Fig. 4), the subunits of the CSC are likely to contribute to the connection between the baseplate and RS2 and might also connect the baseplate to RS3S (Heuser et al. 2009; Heuser et al. 2012b). The DRC linker begins approximately at protofilament A4 and extends toward the B tubule of the neighboring outer doublet (Fig. 5). It is a very large structure $(\sim 1.2 \mathrm{MDa})$ and contains several subdomains. One subdomain is a protrusion that connects the DRC to the outer dynein arms known as OID2 (Fig. 4). Chemical cross-linking studies suggest that this linker involves a direct interaction between DRC4 and IC2 (IC69) of the outer-arm dynein (Oda et al. 2013). A second subdomain is a protrusion that connects the DRC to dynein $g$ (IA5). This protrusion is defective in the $d r c 3$ ( fap134) mutant (Awata et al. 2015). As the DRC linker reaches across the interdoublet 


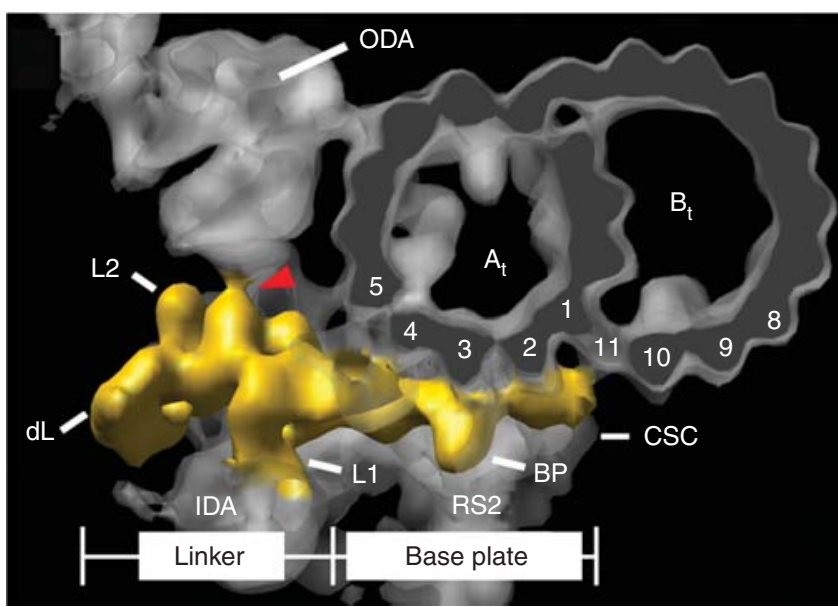

Figure 5. Cryo-electron tomography (cryo-ET) reconstruction of the nexin-dynein regulatory complex (N-DRC). This image shows the $\mathrm{N}$-DRC in yellow, as viewed from the distal end of a 96-nm axoneme repeat in cross section. A single outer doublet is shown here, with a subset of protofilaments numbered in the A tubule $\left(A_{t}\right)$ and $B$ tubule $\left(B_{t}\right)$. The outer dynein arms (ODA) are shown in gray at the top left, and one of the inner dynein arms (IDA) is shown in gray at the bottom left. The baseplate of the N-DRC is attached to protofilament 11 on the $\mathrm{B}$ tubule and wraps around the underside of the A tubule until protofilament 4. The linker of the N-DRC extends from the surface of the A tubule toward the neighboring outer doublet, and it also contacts both the IDA (at L1) and the ODA (red arrowhead). Radial spoke 2 (RS2) attaches to the A tubule just behind the N-DRC. The calmodulin- and spoke-associated complex (CSC) sits at the base of RS2 and wraps around the N-DRC to the next RS3 or RS3S, which is not shown here. The precise boundaries among the subunits of RS2, the CSC, and the base of the DRC are not well defined. (Adapted from Heuser et al. 2009.)

space, it branches into two other subdomains-proximal and distal (Figs. 4 and 5). Interestingly, both the proximal and distal subdomains make contact with protofilament B8 of the neighboring $\mathrm{B}$ tubule. These observations suggest that the alignment of the DRC with the neighboring outer doublet is quite precise, as compared with the dynein arms, which contact several different B-tubule protofilaments (Lin et al. 2014a).

Nearly all of the DRC linker is missing in $p f 2$ and sup-pf3 axonemes, and a major portion is also missing in $p f 3$. Given that the DRC linker is the only structure that interconnects the outer doublets besides the dynein arms, the obvious conclusion is that the DRC is part of the nexin link (Heuser et al. 2009). However, given that most investigators have assumed that the nexin link is essential for axoneme integrity, it was surprising that none of the existing $d r c$ mutants showed any obvious defects in the arrangement of outer doublet microtubules. These observations suggested that other components remaining in the $d r c$ mutants are sufficient to maintain axoneme integrity in vivo.
Interestingly, mutations in two new genes that affect the assembly of DRC subunits have been identified recently in dogs, zebrafish, and humans (Becker-Heck et al. 2011; Merveille et al. 2011). These mutations disrupt the genes encoding two highly conserved, coiled-coil proteins known as CCDC39 and CCDC40 in vertebrates. Although the biochemical defects have not been well characterized in vertebrates, both mutants have obvious structural defects in the assembly of IDAs and the DRC. In addition, mutations in both genes are associated with varying degrees of microtubule disorganization within the axoneme. The Chlamydomonas orthologs of CCDC40 and CCDC39-FAP172 and FAP59-were recently identified as the defective gene products in $p f 7$ and $p f 8$, respectively (Lin et al. 2015). Loss of these proteins in $p f 7$ and $p f 8$ mutant axonemes disrupts the assembly of several IDAs and N-DRC subunits (Lin et al. 2015). By tagging the amino- and carboxy-terminal domains of FAP172 and FAP59 and transforming the tagged constructs into the $p f 7$ and $p f 8$ mutants, Oda and colleagues (Oda et al. 2014a) determined that these proteins form a long, extended structure that serves as a molecular "ruler" to define the $96-\mathrm{nm}$ repeat and mark the locations of specific binding sites for several IDAs, the RSs, and the N-DRC (Oda et al. 2014a). Additional work is needed to determine how each of these complexes is docked to its appropriate location.

\subsection{DRC Subunit Composition and Function}

Analysis by one-dimensional (1D) and two-dimensional (2D) gel electrophoresis identified seven polypeptides that are missing to varying degrees in the $d r c$ mutants ( $\mathrm{Ta}$ ble 4) (Huang et al. 1982; Piperno et al. 1992; Piperno et al. 1994). DRC4 was first identified by rescue of a $p f 2$ mutation that had been tagged by plasmid insertion, and corresponds to a highly conserved, coiled-coil protein of $\sim 55 \mathrm{kDa}$, which is ubiquitous in organisms with motile axonemes (Rupp and Porter 2003). As mentioned above, pf2 mutants in Chlamydomonas have defects in the assembly of the DRC linker (Fig. 5). Transformation with both wild-type and epitope-tagged versions of PF2/DRC4 restores motility and all of the missing polypeptides and structures (Rupp and Porter 2003; Heuser et al. 2009; Bower et al. 2013). These results suggest that DRC4 plays a crucial role in the assembly of the DRC linker domain. This hypothesis is further supported by studies on an unusual DRC4 mutation known as sup-pf3 (Bower et al. 2013). The sup-pf3 mutant is the result of transposon insertion into the DRC4 gene, resulting in alternate splicing and in-frame deletions within the DRC4 coding sequence. The truncated DRC4 subunits assemble into the axoneme, but most of the linker polypeptides are still missing 
R. Viswanadha et al.

Table 4. Nexin-dynein regulatory complex (N-DRC) proteins

\begin{tabular}{|c|c|c|c|c|c|}
\hline $\begin{array}{l}\text { Chlamydomonas } \\
\text { name }\end{array}$ & $\begin{array}{l}\mathrm{MW} \\
(\mathrm{kDa})\end{array}$ & Motifs & $\begin{array}{l}\text { Missing or reduced in } \\
\text { mutant }\end{array}$ & $\begin{array}{c}\text { Homo } \\
\text { sapiens }\end{array}$ & References \\
\hline DRC1 & 79 & $\begin{array}{l}\text { Coiled- } \\
\text { coil }\end{array}$ & $p f 3$ & CCDC164 & Wirschell et al. 2013 \\
\hline DRC2 (FAP250) & 65 & $\begin{array}{l}\text { Coiled- } \\
\text { coil }\end{array}$ & $p f 3$, ida6 & CCDC65 & $\begin{array}{l}\text { Lin et al. 2011; Bower et al. 2013; Austin-Tse et al. } \\
2013\end{array}$ \\
\hline DRC3 (FAP134) & 60 & LRR & $p f 2$, sup-pf3, drc3 & LRRC48 & Lin et al. 2011; Bower et al. 2013; Awata et al. 2015 \\
\hline DRC4 (PF2) & 55 & $\begin{array}{l}\text { Coiled- } \\
\text { coil }\end{array}$ & $p f 2$, sup-pf3 & GAS11 & Rupp and Porter 2003 \\
\hline DRC5 (FAP155) & 43 & LRR & all except $d r c 3$ & TCTE1 & Lin et al. 2011; Bower et al. 2013 \\
\hline DRC6 (FAP169) & 28 & - & all except $d r c 3$ & FBXL13 & Lin et al. 2011; Bower et al. 2013 \\
\hline DRC7 (FAP50) & 177 & LRR, TGL & all except sup-pf4, drc3 & CCDC135 & Lin et al. 2011; Bower et al. 2013 \\
\hline DRC8 (FAP200) & 19 & EF hand & all except sup-pf4, $d r c 3$ & EFCBD2 & Bower et al. 2013 \\
\hline DRC9 (FAP122) & 46 & IQ & all except sup-pf4, $d r c 3$ & IQCG & Bower et al. 2013 \\
\hline DRC10 (FAP84) & 41 & IQ & all except sup-pf4, $d r c 3$ & IQCD & Bower et al. 2013 \\
\hline DRC11 (FAP82) & 95 & AAA, IQ & all except sup-pf4, $d r c 3$ & IQCA1 & Bower et al. 2013 \\
\hline
\end{tabular}

MW, molecular weight; AAA, “ATPases associated with diverse cellular activities” motif; EF hand, $\mathrm{Ca}^{2+}$-binding motif; IQ, IQ calmodulin-binding motif; LRR, leucine-rich repeat; DRC, dynein regulatory complex; TGL, transglutaminase-like.

(Table 4). The structures that remain are the baseplate and a small domain at the base of the linker region, close to the A tubule (Heuser et al. 2009). These observations and other studies indicating that DRC4 can be chemically crosslinked to the outer dynein arm subunit IC2 (Oda et al. 2013) have led to a model that predicted that a portion of DRC4 is located close to the base of the DRC linker. Tagging of DRC4 subunits at several sites followed by cryo-ET localization of tagged DRC4 constructs has recently revealed that DRC4 is an elongated coiled-coil protein that extends from one end of the baseplate to the end of the linker region (Oda et al. 2015; Song et al. 2015).

Studies of DRC4 orthologs in other species indicate that this subunit plays a conserved role in ciliary and flagellar motility. For instance, RNA interference (RNAi)-mediated knockdown of a trypanosome ortholog known as trypanin has shown that this protein is required for optimal flagellar motility and is essential for viability of the bloodstream form of the parasite that causes sleeping sickness (Ralston and Hill 2006). DRC4 is also essential for ciliary motility in vertebrates. Known as GAS11 in humans and GAS8 in mice and zebrafish, the vertebrate ortholog was first described as a gene in mouse fibroblasts whose expression was stimulated by serum starvation and growth arrest. These observations prompted several investigators to search for a role for DRC4/Gas8/Gas11 in primary cilia, but thus far the evidence has been inconclusive (reviewed in Porter 2012). However, knockdown of GAS8 in zebrafish results in hydrocephaly, neural tube cell death, left-right axis defects, and defects in otolith biogenesis-all phenotypes associated with defects in ciliary motility (Colantonio et al. 2009). Mutations in DRC4/GAS11 have recently been found in three patients with primary ciliary dyskinesia (PCD) (Olbrich et al. 2015).

Mutations in DRC1 and DRC2 have also recently been identified in Chlamydomonas and human patients with PCD (Austin-Tse et al. 2013; Wirschell et al. 2013). In Chlamydomonas, DRC1 is the PF3 gene product, and the pf3 mutation is a premature stop codon that truncates the DRC1 protein after six amino acids (Wirschell et al. 2013). The mutant DRC1 polypeptide fails to assemble into the axoneme, and most of the other DRC subunits are also missing or reduced (Table 4). Consistent with these observations, the $p f 3$ mutant fails to assemble most of the baseplate and DRC linker (Fig. 5) (Heuser et al. 2009). The defects impact connections to other structures within this region. For example, several of the inner-arm dyneins most closely associated with RS2 are reduced (Gardner et al. 1994; Heuser et al. 2009; Wirschell et al. 2013). In addition, interactions between the base of RS2 and the CSC appear to be disrupted (Fig. 4) (Heuser et al. 2009; Dymek et al. 2011; Heuser et al. 2012b). Tagging of DRC1 followed by cryo-ET localization of $D R C 1$ constructs has shown that $\mathrm{DRC1}$ also extends from one end of the baseplate to the end of the linker region (Oda et al. 2015).

Similar to DRC4, DRC1 is a highly conserved, coiledcoil protein that appears to be ubiquitous in organisms with motile axonemes (Bower et al. 2013; Wirschell et al. 2013). The vertebrate ortholog is known as CCDC164, and mutations in this sequence have been linked to PCD in at least three families (Wirschell et al. 2013). The ccdc164 mutations reduce the amplitude of the ciliary beat in isolated respiratory cilia and also disrupt the assembly of the nexin links and at least two DRC subunits. Interestingly, 
however, the organization of microtubules within the axoneme is relatively normal, and CCDC39 is also present, similar to the Chlamydomonas pf 3 mutant. These observations indicate that the phenotypes of typical $d r c$ mutations in human patients can be extremely subtle and potentially easy to overlook by conventional transmission electron microscopy (TEM) in the absence of careful analysis of the ciliary waveforms (Wirschell et al. 2013).

The remaining DRC subunits have been identified by a combination of 2D gel electrophoresis, immunoprecipitation, labeling by a technique known as "isobaric tag for relative and absolute quantitation" (iTRAQ) and mass spectrometry (Table 4) (Lin et al. 2011; Bower et al. 2013). The efforts have resulted in the characterization of nine additional subunits, although it is highly likely that there are others yet to be identified.

DRC2 is another highly conserved coiled-coil polypeptide known as FAP250 in Chlamydomonas, CCDC65 in vertebrates, and CMF70 in trypanosomes (Kabututu et al. 2010; Austin-Tse et al. 2013). Its sequence is closely related to DRC1/CCDC164, and the two proteins appear to interact based on their coassembly in $p f 2$, sup- $p f 3$, and sup-pf4 and their absence in pf3 and sup-pf5 (Table 4). Recent work has identified DRC2 as the mutant gene product of the IDA6 locus (Austin-Tse et al. 2013). The ida6 mutant was first identified as an inner-arm motility mutant lacking dynein $e$, similar to $p f 3$ (Kato 1993; Gardner et al. 1994). Later studies revealed that the tektin polypeptide, thought to be a component of the A-tubule protofilament ribbon, is also significantly reduced in ida6, $p f 3$, and suppf5 (Yanagisawa and Kamiya 2004). These observations prompted a reevaluation of the ida6 mutant by thin-section TEM and image averaging. This approach revealed that ida6 also has significant defects in DRC structure that can be rescued by transformation with wild-type DRC2 (Austin-Tse et al. 2013). Tagging and localization of DRC2 subunits has further shown that DRC2 also extends from the baseplate to the linker region, similar to DRC1 and DRC4 (Oda et al. 2015). Additional work is needed to characterize the full extent of the structural and biochemical defects in ida6, but studies in other species have confirmed that DRC2 orthologs play a conserved role in motility. Knockdown of CCDC65 and CMF70 in zebrafish and trypanosomes alters the ciliary and flagellar beat patterns, and mutations in CCDC65 have been linked to PCD in at least three families (Kabututu et al. 2010; Austin-Tse et al. 2013; Horani et al. 2013).

DRC3 is known as FAP134 in Chlamydomonas and the leucine-rich repeat (LRR) protein LRRC48 in vertebrates (Lin et al. 2011; Bower et al. 2013). A drc3 mutant has been identified in Chlamydomonas (Lechtreck et al. 2009). Loss of DRC3 disrupts the assembly of IDA $g$, but it has minimal effects on the assembly of other N-DRC subunits (Awata et al. 2015). However, DRC3 appears to be closely associated with DRC4, based on its assembly with truncated DRC4 subunits in sup-pf3 and its absence in the DRC4null mutant $p f 2$ (Bower et al. 2013; Alford et al. 2016). Tagging of DRC3 and localization by cryo-ET has shown that DRC3 is an important component of the protrusion that connects the linker region to IDA $g$ (Song et al. 2015).

DRC5 (FAP155) and DRC6 (FAP169) are subunits associated with the distal region of the linker domain that makes contact with the neighboring B tubule (Figs. 4 and 5). This conclusion is based on the structural and biochemical phenotypes of the sup-pf4 mutant, which lacks only two polypeptides and a small portion of the distal linker (Huang et al. 1982; Piperno et al. 1992; Gardner et al. 1994; Piperno et al. 1994; Heuser et al. 2009). More recent work has shown that the sup-pf4 mutation is a large deletion that removes DRC5 and two neighboring genes (Lin et al. 2011). The loss of DRC5 and DRC6 partially destabilizes the isolated DRC in sup-pf4 extracts, but transformation with DRC5-HA rescues the mutant and restabilizes the DRC (Bower et al. 2013). It is not known which subunit makes direct contact with the B tubule. The carboxyl terminus of DRC5 is located near the base of the linker, as determined by cryo-ET localization of a tagged construct (Oda et al. 2015), but the amino terminus has not yet been localized. However, the DRC5 (FAP155) sequence is a LRR protein that is significantly more highly conserved in other species with motile axonemes than the DRC6 (FAP169) sequence, and so DRC5 (FAP155) seems to be the more likely candidate (Bower et al. 2013). No mutations have been described in other species, but, given the subtle motility defects observed with sup-pf4 in Chlamydomonas, such mutations might be difficult to detect.

At $\sim 177 \mathrm{kDa}$, DRC7 is the largest known subunit of the DRC, and it contains several conserved domains, including LRR sequences in the amino-terminal third of the polypeptide. However, the most highly conserved region is the carboxy-terminal third of the polypeptide, which contains the recently identified transglutaminaselike (TGL) peptidase domain (Zhang and Aravind 2012). This motif is conserved in both the Drosophila ortholog CG34110 and the vertebrate ortholog CCDC135, and it is predicted to bind tightly to glutamylated proteins. Because DRC7/FAP50 appears to be a major component of the linker region, it is tempting to speculate that the TGL domain facilitates interactions with glu-tubulin residues on the B tubule. Studies of glutamylation-defective mutants (tpg1,tpg2) in Chlamydomonas have indicated that microtubule sliding is altered in these strains (Kubo et al. 2010). Although no DRC7/FAP50 mutations have been identified in Chlamydomonas, a mutation in the Drosophila ortholog 
CG34110 known as lost boys (lobo) is associated with defects in the movement of sperm in the female reproductive tract (Yang et al. 2011). This movement is a $\mathrm{Ca}^{2+}$-regulated process that requires changes in the flagellar waveform and in the direction of wave propagation. As no $\mathrm{Ca}^{2+}$-binding domain has been identified in DRC7, it seems likely that some other $\mathrm{Ca}^{2+}$-binding proteins are also involved in this process.

The DRC8-DRC11 subunits contain either $\mathrm{Ca}^{2+}$ - or CaM-binding domains, suggesting that they contribute to one of the many pathways that modulate flagellar motility in response to changes in intracellular $\mathrm{Ca}^{2+}$ (Bower et al. 2013). DRC11 also contains an AAA domain that could potentially mediate nucleotide-sensitive conformational changes. These subunits are reduced in all of the $d r c \mathrm{mu}-$ tants except sup-pf4, but little is yet known about their specific interactions or locations within the larger structure of the N-DRC (Bower et al. 2013). Additional work is needed to understand how they fit within the network of mechanochemical signals transmitted from the RS heads to the RS stalk, from the RS stalk to the CSC and N-DRC, and from the N-DRC to multiple inner-arm dyneins. The isolation and identification of selective mutations in all of the $\mathrm{N}$-DRC subunits will be an essential next step for the dissection of this complex regulatory hub.

The presence of several DRC subunits in organisms with motile axonemes that lack the CP, RS, and IDAs (Bower et al. 2013) also suggests a more basic function for the NDRC beyond the higher-order control of waveform provided by the RS/CP system. An intriguing possibility is that the N-DRC plays a crucial role in maintaining the outerdoublet alignment in motile $9+0$ axonemes. This hypothesis is consistent with the presence of the nexin links in extracted ciliary remnants first described more than 40 years ago and more recent work indicating that loss of the N-DRC increases the splaying of outer doublets in isolated, ATP-treated axonemes (Bower et al. 2013; Alford et al. 2016). The further study of motile $9+0$ axonemes might shed additional light on the functions of the N-DRC.

\section{CONCLUSION}

Comparative genomics and proteomics have revealed that ciliary and flagellar axonemes contain more than 500 proteins. Although significant progress has been made in assigning many of these proteins to specific structures, more than half of these proteins are still uncharacterized. Recent work featured in this review has revealed two important regulatory hubs within the 96-nm repeat that interconnect several of the most well-characterized axoneme components. Additional work using specifically tagged subunits is still needed to understand how these different structures interact. New biophysical tools, including optical approaches, are also required so that we can test the various models for how the axonemal subcomplexes work together in vivo to generate motility.

\section{ACKNOWLEDGMENTS}

This work was supported by a predoctoral fellowship from the American Heart Association (AHA-11PRE7440043 to R.V.) and grants from the National Institutes of Health (NIH) (GM-051173 to W.S.S.; GM-055667 to M.E.P.).

\section{REFERENCES}

Ahmed NT, Mitchell DR. 2005. ODA16p, a Chlamydomonas flagellar protein needed for dynein assembly. Mol Biol Cell 16: 5004-5012.

Alford LM, Stoddard D, Li JH, Hunter EL, Tritschler D, Bower R, Nicastro D, Porter ME, Sale WS. 2016. The nexin link and B-tubule glutamylation maintain the alignment of outer doublets in the ciliary axoneme. Cytoskeleton 73: 331-340.

Austin-Tse C, Halbritter J, Zariwala MA, Gilberti RM, Gee HY, Hellman N, Pathak N, Liu Y, Panizzi JR, Patel-King RS, et al. 2013. Zebrafish ciliopathy screen plus human mutational analysis identifies C21orf59 and CCDC65 defects as causing primary ciliary dyskinesia. Am J Hum Genet 93: 672-686.

Awata J, Song K, Lin J, King SM, Sanderson MJ, Nicastro D, Witman GB. 2015. DRC3 connects the N-DRC to dynein $g$ to regulate flagellar waveform. Mol Biol Cell 26: 2788-2800.

Barber CF, Heuser T, Carbajal-Gonzalez BI, Botchkarev VV Jr, Nicastro D. 2012. Three-dimensional structure of the radial spokes reveals heterogeneity and interactions with dyneins in Chlamydomonas flagella. Mol Biol Cell 23: 111-120.

Bayly PV, Lewis BL, Kemp PS, Pless RB, Dutcher SK. 2010. Efficient spatiotemporal analysis of the flagellar waveform of Chlamydomonas reinhardtii. Cytoskeleton 67: 56-69.

Becker-Heck A, Zohn IE, Okabe N, Pollock A, Lenhart KB, SullivanBrown J, McSheene J, Loges NT, Olbrich H, Haeffner K, et al. 2011. The coiled-coil domain containing protein CCDC40 is essential for motile cilia function and left-right axis formation. Nat Genet 43: 7984.

Bower R, Vanderwaal K, O’Toole E, Fox L, Perrone C, Mueller J, Wirschell M, Kamiya R, Sale WS, Porter ME. 2009. IC138 defines a sub-domain at the base of the I1 dynein that regulates microtubule sliding and flagellar motility. Mol Biol Cell 20: 3055-3063.

Bower R, Tritschler D, Vanderwaal K, Perrone CA, Mueller J, Fox L, Sale WS, Porter ME. 2013. The N-DRC forms a conserved biochemical complex that maintains outer doublet alignment and limits microtubule sliding in motile axonemes. Mol Biol Cell 24: $1134-1152$

Bozkurt HH, Woolley DM. 1993. Morphology of nexin links in relation to interdoublet sliding in the sperm flagellum. Cell Motil Cytoskeleton 24: $109-118$.

Brokaw CJ. 1985. Computer simulation of flagellar movement. VI. Simple curvature-controlled models are incompletely specified. Biophys $J$ 48: $633-642$.

Brokaw CJ. 1989. Direct measurements of sliding between outer doublet microtubules in swimming sperm flagella. Science 243: 1593-1596.

Brokaw CJ. 2009. Thinking about flagellar oscillation. Cell Motil Cytoskeleton 66: 425-436.

Brokaw CJ, Kamiya R. 1987. Bending patterns of Chlamydomonas flagella: IV. Mutants with defects in inner and outer dynein arms indicate differences in dynein arm function. Cell Motil Cytoskeleton 8: $68-75$. 
Bui KH, Ishikawa T. 2013. 3D structural analysis of flagella/cilia by cryoelectron tomography. Methods Enzymol 524: 305-323.

Bui KH, Sakakibara H, Movassagh T, Oiwa K, Ishikawa T. 2009. Asymmetry of inner dynein arms and inter-doublet links in Chlamydomonas flagella. J Cell Biol 186: 437-446.

Bui KH, Yagi T, Yamamoto R, Kamiya R, Ishikawa T. 2012. Polarity and asymmetry in the arrangement of dynein and related structures in the Chlamydomonas axoneme. J Cell Biol 198: 913-925.

Casey DM, Inaba K, Pazour GJ, Takada S, Wakabayashi K, Wilkerson CG, Kamiya R, Witman GB. 2003. Dc3, the 21-kDa subunit of the outer arm-docking complex (ODA-DC), is a novel EF-hand protein important for the assembly of both the outer arm and ODA-DC. Mol Biol Cell 14: 3650-3663.

Carbajal-Gonzalez BI, Heuser T, Fu X, Lin J, Smith BW, Mitchell DR, Nicastro D. 2013. Conserved structural motifs in the central pair complex of eukaryotic flagella. Cytoskeleton 70: 101-120.

Colantonio JR, Vermot J, Wu D, Langenbacher AD, Fraser S, Chen JN, Hill KL. 2009. The dynein regulatory complex is required for ciliary motility and otolith biogenesis in the inner ear. Nature 457: 205-209.

Curry AM, Williams BD, Rosenbaum JL. 1992. Sequence analysis reveals homology between two proteins of the flagellar radial spoke. Mol Cell Biol 12: 3967-3977.

Dean AB, Mitchell DR. 2013. Chlamydomonas ODA10 is a conserved axonemal protein that plays a unique role in outer dynein arm assembly. Mol Biol Cell 24: 3689-3696.

DiBella LM, Benashski SE, Tedford HW, Harrison A, Patel-King RS, King SM. 2001. The Tctex1/Tctex2 class of dynein light chains. Dimerization, differential expression, and interaction with the LC8 protein family. J Biol Chem 276: 14366-14373.

DiBella LM, Sakato M, Patel-King RS, Pazour GJ, King SM. 2004a. The LC7 light chains of Chlamydomonas flagellar dyneins interact with components required for both motor assembly and regulation. Mol Biol Cell 15: 4633-4646.

DiBella LM, Smith EF, Patel-King RS, Wakabayashi K, King SM. 2004b. A novel Tctex2-related light chain is required for stability of inner dynein arm I1 and motor function in the Chlamydomonas flagellum. J Biol Chem 279: 21666-21676.

DiBella LM, Gorbatyuk O, Sakato M, Wakabayashi K, Patel-King RS, Pazour GJ, Witman GB, King SM. 2005. Differential light chain assembly influences outer arm Dynein motor function. Mol Biol Cell 16: 5661-5674.

Duriez B, Duquesnoy P, Escudier E, Bridoux AM, Escalier D, Rayet I, Marcos E, Vojtek AM, Bercher JF, Amselem S. 2007. A common variant in combination with a nonsense mutation in a member of the thioredoxin family causes primary ciliary dyskinesia. Proc Natl Acad Sci 104: 3336-3341.

Dutcher SK. 2014. The awesome power of dikaryons for studying flagella and basal bodies in Chlamydomonas reinhardtii. Cytoskeleton 71: 79-94.

Dymek EE, Smith EF. 2007. A conserved CaM- and radial spoke associated complex mediates regulation of flagellar dynein activity. J Cell Biol 179: $515-526$.

Dymek EE, Heuser T, Nicastro D, Smith EF. 2011. The CSC is required for complete radial spoke assembly and wild-type ciliary motility. Mol Biol Cell 22: 2520-2531.

Elam CA, Wirschell M, Yamamoto R, Fox LA, York K, Kamiya R, Dutcher SK, Sale WS. 2011. An axonemal PP2A B-subunit is required for PP2A localization and flagellar motility. Cytoskeleton 68: 363-372.

Freshour J, Yokoyama R, Mitchell DR. 2007. Chlamydomonas flagellar outer row dynein assembly protein ODA7 interacts with both outer row and I1 inner row dyneins. J Biol Chem 282: 5404-5412.

Gaillard AR, Diener DR, Rosenbaum JL, Sale WS. 2001. Flagellar radial spoke protein 3 is an A-kinase anchoring protein (AKAP). J Cell Biol 153: $443-448$.

Gardner LC, O’Toole E, Perrone CA, Giddings T, Porter ME. 1994. Components of a "dynein regulatory complex" are located at the junction between the radial spokes and the dynein arms in Chlamydomonas flagella. J Cell Biol 127: 1311-1325.

Gibbons IR. 1963. Studies on the protein components of cilia from Tetrahymena pyriformis. Proc Natl Acad Sci 50: 1002-1010.

Gibbons BH, Gibbons IR. 1972. Flagellar movement and adenosine triphosphatase activity in sea urchin sperm extracted with triton X-100.J Cell Biol 54: 75-97.

Gibbons BH, Gibbons IR. 1973. The effect of partial extraction of dynein arms on the movement of reactivated sea-urchin sperm. J Cell Sci 13: 337-357.

Gokhale A, Wirschell M, Sale WS. 2009. Regulation of dynein-driven microtubule sliding by the axonemal protein kinase CK1 in Chlamydomonas flagella. J Cell Biol 186: 817-824.

Goodenough UW, Heuser JE. 1982. Substructure of the outer dynein arm. J Cell Biol 95: 798-815.

Goodenough UW, Heuser JE. 1985. Substructure of inner dynein arms, radial spokes, and the central pair/projection complex of cilia and flagella. J Cell Biol 100: 2008-2018.

Habermacher G, Sale WS. 1997. Regulation of flagellar dynein by phosphorylation of a $138-\mathrm{kD}$ inner arm dynein intermediate chain. J Cell Biol 136: $167-176$.

Harris EH. 2009. The Chlamydomonas sourcebook: Introduction to Chlamydomonas and its laboratory use. Academic, Oxford.

Harrison A, Olds-Clarke P, King SM. 1998. Identification of the $t$ complex-encoded cytoplasmic dynein light chain tctexl in inner arm I1 supports the involvement of flagellar dyneins in meiotic drive. J Cell Biol 140: 1137-1147.

Hayashi S, Shingyoji C. 2008. Mechanism of flagellar oscillation-bending-induced switching of dynein activity in elastase-treated axonemes of sea urchin sperm. J Cell Sci 121: 2833-2843.

Hendrickson TW, Perrone CA, Griffin P, Wuichet K, Mueller J, Yang P, Porter ME, Sale WS. 2004. IC138 is a WD-repeat dynein intermediate chain required for light chain assembly and regulation of flagellar bending. Mol Biol Cell 12: 5431-5442.

Heuser T, Raytchev M, Krell J, Porter ME, Nicastro D. 2009. The dynein regulatory complex is the nexin link and a major regulatory node in cilia and flagella. J Cell Biol 187: 921-933.

Heuser T, Barber CF, Lin J, Krell J, Rebesco M, Porter ME, Nicastro D. 2012a. Cryoelectron tomography reveals doublet-specific structures and unique interactions in the I1 dynein. Proc Natl Acad Sci 109: E2067-E2076.

Heuser T, Dymek EE, Lin J, Smith EF, Nicastro D. 2012b. The CSC connects three major axonemal complexes involved in dynein regulation. Mol Biol Cell 23: 3143-3155.

Hirono M, Uryu S, Ohara A, Kato-Minoura T, Kamiya R. 2003. Expression of conventional and unconventional actins in Chlamydomonas reinhardtii upon deflagellation and sexual adhesion. Eukaryot Cell 2: 486-493.

Hom EF, Witman GB, Harris EH, Dutcher SK, Kamiya R, Mitchell DR, Pazour GJ, Porter ME, Sale WS, Wirschell M, et al. 2011. A unified taxonomy for ciliary dyneins. Cytoskeleton 68: 555-565.

Hoops HJ, Witman GB. 1983. Outer doublet heterogeneity reveals structural polarity related to beat direction in Chlamydomonas flagella. J Cell Biol 97: 902-908.

Horani A, Brody SL, Ferkol TW, Shoseyov D, Wasserman MG, Ta-shma A, Wilson KS, Bayly PV, Amirav I, Cohen-Cymberknoh M, et al. 2013. CCDC65 mutation causes primary ciliary dyskinesia with normal ultrastructure and hyperkinetic cilia. PLoS ONE 8: e72299.

Huang B, Piperno G, Luck DJ. 1979. Paralyzed flagella mutants of Chlamydomonas reinhardtii. Defective for axonemal doublet microtubule arms. J Biol Chem 254: 3091-3099.

Huang B, Ramanis Z, Luck DJ. 1982. Suppressor mutations in Chlamydomonas reveal a regulatory mechanism for flagellar function. Cell 28: $115-124$.

Huang B, Watterson DM, Lee VD, Schibler MJ. 1988. Purification and characterization of a basal body-associated $\mathrm{Ca}^{2+}$-binding protein. J Cell Biol 107: 121-131. 
R. Viswanadha et al.

Ichikawa M, Saito K, Yanagisawa H, Yagi T, Kamiya R, Kushida Y, Nakano K, Numata O, Toyoshima YY. 2014. Axonemal dynein light chain 1 locates at the microtubule-binding domain of the $\gamma$ heavy chain. Mol Biol Cell 26: 4236-4247.

Ikeda K, Yamamoto R, Wirschell M, Yagi T, Bower R, Porter ME, Sale WS, Kamiya R. 2009. A novel ankyrin-repeat protein interacts with the regulatory proteins of inner arm dynein $\mathrm{f}$ (I1) of Chlamydomonas reinhardtii. Cell Motil Cytoskeleton 66: 448-456.

Kabututu ZP, Thayer M, Melehani JH, Hill KL. 2010. CMF70 is a subunit of the dynein regulatory complex. J Cell Sci 123: 3587-3595.

Kamiya R. 1988. Mutations at twelve independent loci result in absence of outer dynein arms in Chlamydomonas reinhardtii. J Cell Biol 107: 2253-2258.

Kamiya R. 1995. Exploring the function of inner and outer dynein arms with Chlamydomonas mutants. Cell Motil Cytoskeleton 32: 98-102.

Kamiya R, Okamoto M. 1985. A mutant of Chlamydomonas reinhardtii that lacks the flagellar outer dynein arm but can swim. J Cell Sci 74: $181-191$.

Kamiya R, Yagi T. 2014. Functional diversity of axonemal dyneins as assessed by in vitro and in vivo motility assays of Chlamydomonas mutants. Zoolog Sci 31: 633-644.

Kamiya R, Kurimoto E, Muto E. 1991. Two types of Chlamydomonas flagellar mutants missing different components of inner-arm dynein. J Cell Biol 112: 441-447.

Kato T, Kagami O, Yagi T, Kamiya R. 1993. Isolation of two species of Chlamydomonas reinhardtii flagellar mutants, ida5 and ida6, that lack a newly identified heavy chain of the inner dynein arm. Cell Struct Funct 18: 371-377.

Kato-Minoura T, Hirono M, Kamiya R. 1997. Chlamydomonas innerarm dynein mutant, ida5, has a mutation in an actin-encoding gene. J Cell Biol 137: 649-656.

Kikkawa M. 2013. Big steps toward understanding dynein. J Cell Biol 202: $15-23$.

King SM. 2010. Sensing the mechanical state of the axoneme and integration of $\mathrm{Ca}^{2+}$ signaling by outer arm dynein. Cytoskeleton 67: 207213.

King SM. 2012. Dyneins: Structure, biology and disease. Academic, New York.

King SM. 2013. A solid-state control system for dynein-based ciliary/ flagellar motility. J Cell Biol 201: 173-175.

King SJ, Dutcher SK. 1997. Phosphoregulation of an inner dynein arm complex in Chlamydomonas reinhardtii is altered in phototactic mutant strains. J Cell Biol 136: 177-191.

King SM, Kamiya R. 2009. Axonemal dyneins: Assembly, structure, and force generation. In The Chlamydomonas sourcebook: Cell motility and behavior (ed. Witman GB), pp. 131-208. Academic, Oxford.

King SM, Patel-King RS. 1995. The $M_{\mathrm{r}}=8,000$ and 11,000 outer arm dynein light chains from Chlamydomonas flagella have cytoplasmic homologues. J Biol Chem 270: 11445-11452.

King SM, Patel-King RS. 2012. Functional architecture of the outer arm dynein conformational switch. J Biol Chem 287: 3108-3122.

Kotani N, Sakakibara H, Burgess SA, Kojima H, Oiwa K. 2007. Mechanical properties of inner-arm dynein- $\mathrm{f}$ (dynein I1) studied with in vitro motility assays. Biophys J 93: 886-894.

Koutoulis A, Pazour GJ, Wilkerson CG, Inaba K, Sheng H, Takada S, Witman GB. 1997. The Chlamydomonas reinhardtii ODA3 gene encodes a protein of the outer dynein arm docking complex. J Cell Biol 137: 1069-1080.

Kubo T, Yanagisawa HA, Yagi T, Hirono M, Kamiya R. 2010. Tubulin polyglutamylation regulates axonemal motility by modulating activities of inner-arm dyneins. Curr Biol 20: 441-445.

Lechtreck KF, Witman GB. 2007. Chlamydomonas reinhardtii hydin is a central pair protein required for flagellar motility. J Cell Biol 176: 473482.

Lechtreck KF, Luro S, Awata J, Witman GB. 2009. HA-tagging of putative flagellar proteins in Chlamydomonas reinhardtii identifies a novel pro- tein of intraflagellar transport complex B. Cell Motil Cytoskeleton 66: 469-482.

LeDizet M, Piperno G. 1995. The light chain p28 associates with a subset of inner dynein arm heavy chains in Chlamydomonas axonemes. Mol Biol Cell 6: 697-711.

Lin J, Tritschler D, Song K, Barber CF, Cobb JS, Porter ME, Nicastro D. 2011. Building blocks of the nexin-dynein regulatory complex in Chlamydomonas flagella. J Biol Chem 286: 29175-29191.

Lin J, Heuser T, Carbajal-Gonzalez BI, Song K, Nicastro D. 2012. The structural heterogeneity of radial spokes in cilia and flagella is conserved. Cytoskeleton 69: 88-100.

Lin J, Okada K, Raytchev M, Smith MC, Nicastro D. 2014a. Structural mechanism of the dynein power stroke. Nat Cell Biol 16: 479-485.

Lin J, Yin W, Smith MC, Song K, Leigh MW, Zariwala MA, Knowles MR, Ostrowski LE, Nicastro D. 2014b. Cryo-electron tomography reveals ciliary defects underlying human RSPH1 primary ciliary dyskinesia. Nat Commun 5: 5727.

Lin H, Zhang Z, Guo S, Chen F, Kessler JM, Wang YM, Dutcher SK. 2015. A NIMA-related kinase suppresses the flagellar instability associated with the loss of multiple axonemal structures. PLoS Genet 11: e1005508.

Lindemann CB, Mitchell DR. 2007. Evidence for axonemal distortion during the flagellar beat of Chlamydomonas. Cell Motil Cytoskeleton 64: $580-589$.

Lindemann CB, Lesich KA. 2010. Flagellar and ciliary beating: The proven and the possible. J Cell Sci 123: 519-528.

Mastronarde DN, O’Toole ET, McDonald KL, McIntosh JR, Porter ME. 1992. Arrangement of inner dynein arms in wild-type and mutant flagella of Chlamydomonas. J Cell Biol 118: 1145-1162.

Merveille AC, Davis EE, Becker-Heck A, Legendre M, Amirav I, Bataille G, Belmont J, Beydon N, Billen F, Clement A, et al. 2011. CCDC39 is required for assembly of inner dynein arms and the dynein regulatory complex and for normal ciliary motility in humans and dogs. Nat Genet 43: 72-78.

Mitchell DR, Brown KS. 1994. Sequence analysis of the Chlamydomonas $\alpha$ and $\beta$ dynein heavy chain genes. J Cell Sci 107 (Pt 3): 635-644.

Mitchell DR, Brown KS. 1997. Sequence analysis of the Chlamydomonas reinhardtii flagellar alpha dynein gene. Cell Motil Cytoskeleton 37: $120-126$.

Mitchell DR, Kang Y. 1991. Identification of oda6 as a Chlamydomonas dynein mutant by rescue with the wild-type gene. J Cell Biol 113: 835842.

Mitchell DR, Nakatsugawa M. 2004. Bend propagation drives central pair rotation in Chlamydomonas reinhardtii flagella. J Cell Biol 166: 709715.

Mitchell DR, Rosenbaum JL. 1985. A motile Chlamydomonas flagellar mutant that lacks outer dynein arms. J Cell Biol 100: 1228-1234.

Mitchison HM, Schmidts M, Loges NT, Freshour J, Dritsoula A, Hirst RA, O'Callaghan C, Blau H, Al Dabbagh M, Olbrich H, et al. 2012. Mutations in axonemal dynein assembly factor DNAAF3 cause primary ciliary dyskinesia. Nat Genet 44: 381-389.

Morita Y, Shingyoji C. 2004. Effects of imposed bending on microtubule sliding in sperm flagella. Curr Biol 14: 2113-2118.

Movassagh T, Bui KH, Sakakibara H, Oiwa K, Ishikawa T. 2010. Nucleotide-induced global conformational changes of flagellar dynein arms revealed by in situ analysis. Nat Struct Mol Biol 17: 761-767.

Myster SH, Knott JA, O’Toole E, Porter ME. 1997. The Chlamydomonas $D h c 1$ gene encodes a dynein heavy chain subunit required for assembly of the I1 inner arm complex. Mol Biol Cell 8: 607-620.

Myster SH, Knott JA, Wysocki KM, O’Toole E, Porter ME. 1999. Domains in the $1 \alpha$ dynein heavy chain required for inner arm assembly and flagellar motility in Chlamydomonas. J Cell Biol 146: 801-818.

Nicastro D. 2009. Cryo-electron microscope tomography to study axonemal organization. Methods Cell Biol 91: 1-39.

Nicastro D, Schwartz C, Pierson J, Gaudette R, Porter ME, McIntosh JR. 2006. The molecular architecture of axonemes revealed by cryoelectron tomography. Science 313: 944-948. 
Oda T, Kikkawa M. 2013. Novel structural labeling method using cryoelectron tomography and biotin-streptavidin system. J Struct Biol 183: $305-311$.

Oda T, Yagi T, Yanagisawa H, Kikkawa M. 2013. Identification of the outer-inner dynein linker as a hub controller for axonemal dynein activities. Curr Biol 23: 656-664.

Oda T, Yanagisawa H, Kamiya R, Kikkawa M. 2014a. A molecular ruler determines the repeat length in eukaryotic cilia and flagella. Science 346: $857-860$

Oda T, Yanagisawa H, Yagi T, Kikkawa M. 2014b. Mechanosignaling between central apparatus and radial spokes controls axonemal dynein activity. J Cell Biol 204: 807-819.

Oda T, Yanagisawa H, Kikkawa M. 2015. Detailed structural and biochemical characterization of the nexin-dynein regulatory complex. Mol Biol Cell 26: 294-304.

Olbrich H, Cremers C, Loges NT, Werner C, Nielsen KG, Marthin JK, Philipsen M, Wallmeier J, Pennekamp P, Menchen T, et al. 2015. Lossof-function GAS8 mutations cause primary ciliary dyskinesia and disrupt the nexin-dynein regulatory complex. Am J Hum Genet 97: 546-554.

Omran H, Kobayashi D, Olbrich H, Tsukahara T, Loges N, Hagiwara H, Zhang Q, Leblond G, O’Toole E, Hara C, et al. 2008. Ktu/PF13 is required for cytoplasmic pre-assembly of axonemal dyneins. Nature 456: $611-616$

O’Toole ET, Giddings TH Jr., Porter ME, Ostrowski LE. 2012. Computerassisted image analysis of human cilia and Chlamydomonas flagella reveals both similarities and differences in axoneme structure. Cytoskeleton 69: $577-590$.

Owa M, Furuta A, Usukura J, Arisaka F, King SM, Witman GB, Kamiya R, Wakabayashi K. 2014. Cooperative binding of the outer arm-docking complex underlies the regular arrangement of outer arm dynein in the axoneme. Proc Natl Acad Sci 111: 9461-9466.

Patel-King RS, King SM. 2009. An outer arm dynein light chain acts in a conformational switch for flagellar motility. J Cell Biol 186: 283-295.

Patel-King RS, Benashski SE, Harrison A, King SM. 1997. A Chlamydomonas homologue of the putative murine $t$ complex distorter Tctex-2 is an outer arm dynein light chain. J Cell Biol 137: 1081-1090.

Patel-King RS, Gorbatyuk O, Takebe S, King SM. 2004. Flagellar radial spokes contain a $\mathrm{Ca}^{2+}$-stimulated nucleoside diphosphate kinase. Mol Biol Cell 15: 3891-3902.

Pazour GJ, Koutoulis A, Benashski SE, Dickert BL, Sheng H, Patel-King RS, King SM, Witman GB. 1999. LC2, the Chlamydomonas homologue of the $t$ complex-encoded protein Tctex2, is essential for outer dynein arm assembly. Mol Biol Cell 10: 3507-3520.

Perrone CA, Yang P, O’Toole E, Sale WS, Porter ME. 1998. The Chlamydomonas IDA7 locus encodes a $140-\mathrm{kDa}$ dynein intermediate chain required to assemble the I1 inner arm complex. Mol Biol Cell 9: 33513365.

Perrone CA, Myster SH, Bower R, O’Toole ET, Porter ME. 2000. Insights into the structural organization of the I1 inner arm dynein from a domain analysis of the $1 \beta$ dynein heavy chain. Mol Biol Cell 11:22972313.

Pigino G, Bui KH, Maheshwari A, Lupetti P, Diener D, Ishikawa T. 2011. Cryoelectron tomography of radial spokes in cilia and flagella. J Cell Biol 195: 673-687.

Pigino G, Maheshwari A, Bui KH, Shingyoji C, Kamimura S, Ishikawa T. 2012. Comparative structural analysis of eukaryotic flagella and cilia from Chlamydomonas, Tetrahymena, and sea urchins. J Struct Biol 178: 199-206.

Piperno G, Ramanis Z, Smith EF, Sale WS. 1990. Three distinct inner dynein arms in Chlamydomonas flagella: Molecular composition and location in the axoneme. J Cell Biol 110: 379-389.

Piperno G, Mead K, Shestak W. 1992. The inner dynein arms I2 interact with a "dynein regulatory complex" in Chlamydomonas flagella. J Cell Biol 118: 1455-1463.

Piperno G, Mead K, LeDizet M, Moscatelli A. 1994. Mutations in the "dynein regulatory complex" alter the ATP-insensitive binding sites for inner arm dyneins in Chlamydomonas axonemes. J Cell Biol 125: $1109-1117$

Porter ME. 2012. Flagellar motility and the dynein regulatory complex. In Dyneins: Structure, biology and disease (ed. King SM), pp. 337-365. Academic, New York.

Porter ME, Sale WS. 2000. The $9+2$ axoneme anchors multiple inner arm dyneins and a network of kinases and phosphatases that control motility. J Cell Biol 151: F37-F42.

Porter ME, Power J, Dutcher SK. 1992. Extragenic suppressors of paralyzed flagellar mutations in Chlamydomonas reinhardtii identify loci that alter the inner dynein arms. J Cell Biol 118: 1163-1176.

Porter ME, Knott JA, Gardner LC, Mitchell DR, Dutcher SK. 1994. Mutations in the SUP-PF-1 locus of Chlamydomonas reinhardtii identify a regulatory domain in the $\beta$-dynein heavy chain. J Cell Biol 126: 14951507.

Porter ME, Knott JA, Myster SH, Farlow SJ. 1996. The dynein gene family in Chlamydomonas reinhardtii. Genetics 144: 569-585.

Porter ME, Bower R, Knott JA, Byrd P, Dentler W. 1999. Cytoplasmic dynein heavy chain $1 \mathrm{~b}$ is required for flagellar assembly in Chlamydomonas. Mol Biol Cell 10: 693-712.

Ralston KS, Hill KL. 2006. Trypanin, a component of the flagellar dynein regulatory complex, is essential in bloodstream form African trypanosomes. PLoS Pathog 2: e101.

Rupp G, Porter ME. 2003. A subunit of the dynein regulatory complex in Chlamydomonas is a homologue of a growth arrest-specific gene product. J Cell Biol 162: 47-57.

Rupp G, O’Toole E, Gardner LC, Mitchell BF, Porter ME. 1996. The suppf-2 mutations of Chlamydomonas alter the activity of the outer dynein arms by modification of the $\gamma$-dynein heavy chain. J Cell Biol 135: $1853-1865$.

Sakato M, Sakakibara H, King SM. 2007. Chlamydomonas outer arm dynein alters conformation in response to $\mathrm{Ca}^{2+}$. Mol Biol Cell 18: $3620-3634$

Sale WS, Satir P. 1977. Direction of active sliding of microtubules in Tetrahymena cilia. Proc Natl Acad Sci 74: 2045-2049.

Sanders MA, Salisbury JL. 1989. Centrin-mediated microtubule severing during flagellar excision in Chlamydomonas reinhardtii.J Cell Biol 108: $1751-1760$.

Satir P. 1968. Studies on cilia. 3. Further studies on the cilium tip and a "sliding filament" model of ciliary motility. J Cell Biol 39: 77-94.

Satir P, Matsuoka T. 1989. Splitting the ciliary axoneme: Implications for a "switch-point" model of dynein arm activity in ciliary motion. Cell Motil Cytoskeleton 14: 345-358.

Satir P, Heuser T, Sale WS. 2014. A structural basis for how motile cilia beat. Bioscience 64: 1073-1083.

Shingyoji C, Murakami A, Takahashi K. 1977. Local reactivation of Triton-extracted flagella by iontophoretic application of ATP. Nature 265: 269-270

Sivadas P, Dienes JM, St Maurice M, Meek WD, Yang P. 2012. A flagellar A-kinase anchoring protein with two amphipathic helices forms a structural scaffold in the radial spoke complex. J Cell Biol 199: 639651.

Smith EF. 2002. Regulation of flagellar dynein by calcium and a role for an axonemal calmodulin and calmodulin-dependent kinase. Mol Biol Cell 13: 3303-3313.

Smith EF. 2007. Hydin seek: Finding a function in ciliary motility. J Cell Biol 176: 403-404.

Smith EF, Sale WS. 1992a. Regulation of dynein-driven microtubule sliding by the radial spokes in flagella. Science 257: 1557-1559.

Smith EF, Sale WS. 1992b. Structural and functional reconstitution of inner dynein arms in Chlamydomonas flagellar axonemes. J Cell Biol 117: $573-581$.

Smith EF, Yang P. 2004. The radial spokes and central apparatus: Mechano-chemical transducers that regulate flagellar motility. Cell Motil Cytoskeleton 57: 8-17.

Song K, Awata J, Tritschler D, Bower R, Witman GB, Porter ME, Nicastro D. 2015. In situ localization of $\mathrm{N}$ and $\mathrm{C}$ termini of subunits of the 
flagellar nexin-dynein regulatory complex (N-DRC) using SNAP tag and cryo-electron tomography. J Biol Chem 290: 5341-5353.

Stephens RE. 1970. Isolation of nexin-The linkage protein responsible for maintenance of nine-fold configuration of flagellar axonemes. Biol Bull 139: 438.

Summers KE, Gibbons IR. 1971. Adenosine triphosphate-induced sliding of tubules in trypsin-treated flagella of sea-urchin sperm. Proc Natl Acad Sci 68: 3092-3096.

Takada S, Kamiya R. 1994. Functional reconstitution of Chlamydomonas outer dynein arms from $\alpha-\beta$ and $\gamma$ subunits: Requirement of a third factor. J Cell Biol 126: 737-745.

Takada S, Wilkerson CG, Wakabayashi K, Kamiya R, Witman GB. 2002. The outer dynein arm-docking complex: Composition and characterization of a subunit (oda1) necessary for outer arm assembly. Mol Biol Cell 13: 1015-1029.

Toba S, Fox LA, Sakakibara H, Porter ME, Oiwa K, Sale WS. 2011. Distinct roles of $1 \alpha$ and $1 \beta$ heavy chains of the inner arm dynein I1 of Chlamydomonas flagella. Mol Biol Cell 22: 342-353.

VanderWaal KE, Yamamoto R, Wakabayashi K, Fox L, Kamiya R, Dutcher SK, Bayly PV, Sale WS, Porter ME. 2011. bop5 Mutations reveal new roles for the IC138 phosphoprotein in the regulation of flagellar motility and asymmetric waveforms. Mol Biol Cell 22: 2862-2874.

Vasudevan KK, Song K, Alford LM, Sale WS, Dymek EE, Smith EF, Hennessey T, Joachimiak E, Urbanska P, Wloga D, et al. 2015. FAP206 is a microtubule-docking adapter for ciliary radial spoke 2 and dynein c. Mol Biol Cell 26: 696-710.

Viswanadha R, Hunter EL, Yamamoto R, Wirschell M, Alford LM, Dutcher SK, Sale WS. 2014. The ciliary inner dynein arm, I1 dynein, is assembled in the cytoplasm and transported by IFT before axonemal docking. Cytoskeleton 71: 573-586.

Wakabayashi K, King SM. 2006. Modulation of Chlamydomonas reinhardtii flagellar motility by redox poise. J Cell Biol 173: 743-754.

Wargo MJ, Smith EF. 2003. Asymmetry of the central apparatus defines the location of active microtubule sliding in Chlamydomonas flagella. Proc Natl Acad Sci 100: 137-142.

Warner FD. 1976. Ciliary inter-microtubule bridges. J Cell Sci 20: 101114.

Warner FD. 1983. Organization of interdoublet links in Tetrahymena cilia. Cell Motil Cytoskel 3: 321-332.

Warner FD, Satir P. 1974. The structural basis of ciliary bend formation. Radial spoke positional changes accompanying microtubule sliding. $J$ Cell Biol 63: 35-63.

Wickstead B, Gull K. 2007. Dyneins across eukaryotes: A comparative genomic analysis. Traffic 8: 1708-1721.

Wilkerson CG, King SM, Witman GB. 1994. Molecular analysis of the $\gamma$ heavy chain of Chlamydomonas flagellar outer-arm dynein. J Cell Sci 107 (Pt 3): 497-506.

Wilkerson CG, King SM, Koutoulis A, Pazour GJ, Witman GB. 1995. The $78,000-M_{\mathrm{r}}$ intermediate chain of Chlamydomonas outer arm dynein is a WD-repeat protein required for arm assembly. J Cell Biol 129: 169178.

Williams BD, Velleca MA, Curry AM, Rosenbaum JL. 1989. Molecular cloning and sequence analysis of the Chlamydomonas gene coding for radial spoke protein 3: Flagellar mutation $p f-14$ is an ochre allele. J Cell Biol 109: 235-245.

Wirschell M, Pazour G, Yoda A, Hirono M, Kamiya R, Witman GB. 2004. Oda5p, a novel axonemal protein required for assembly of the outer dynein arm and an associated adenylate kinase. Mol Biol Cell 15: 2729-2741.

Wirschell M, Hendrickson T, Sale WS. 2007. Keeping an eye on I1: I1 dynein as a model for flagellar dynein assembly and regulation. Cell Motil Cytoskeleton 64: 569-579.

Wirschell M, Yang C, Yang P, Fox L, Yanagisawa HA, Kamiya R, Witman GB, Porter ME, Sale WS. 2009. IC97 Is a novel intermediate chain of I1 dynein that interacts with tubulin and regulates interdoublet sliding. Mol Biol Cell 20: 3044-3054.

Wirschell M, Olbrich H, Werner C, Tritschler D, Bower R, Sale WS, Loges NT, Pennekamp P, Lindberg S, Stenram U, et al. 2013. The nexindynein regulatory complex subunit DRC1 is essential for motile cilia function in algae and humans. Nat Genet 45: 262-268.

Witman GB, Plummer J, Sander G. 1978. Chlamydomonas flagellar mutants lacking radial spokes and central tubules. Structure, composition, and function of specific axonemal components. J Cell Biol 76: $729-747$.

Woolley DM. 1997. Studies on the eel sperm flagellum. I. The structure of the inner dynein arm complex. J Cell Sci 110: 85-94.

Wu H, Maciejewski MW, Takebe S, King SM. 2005. Solution structure of the Tctex 1 dimer reveals a mechanism for dynein-cargo interactions. Structure 13: 213-223.

Yagi T, Minoura I, Fujiwara A, Saito R, Yasunaga T, Hirono M, Kamiya R. 2005. An axonemal dynein particularly important for flagellar movement at high viscosity. Implications from a new Chlamydomonas mutant deficient in the dynein heavy chain gene DHC9. J Biol Chem 280: $41412-41420$.

Yagi T, Uematsu K, Liu Z, Kamiya R. 2009. Identification of dyneins that localize exclusively to the proximal portion of Chlamydomonas flagella. J Cell Sci 122: 1306-1314.

Yamamoto R, Yanagisawa HA, Yagi T, Kamiya R. 2006. A novel subunit of axonemal dynein conserved among lower and higher eukaryotes. FEBS Lett 580: 6357-6360.

Yamamoto R, Yanagisawa HA, Yagi T, Kamiya R. 2008. Novel 44-kilodalton subunit of axonemal dynein conserved from Chlamydomonas to mammals. Eukaryot Cell 7: 154-161.

Yamamoto R, Hirono M, Kamiya R. 2010. Discrete PIH proteins function in the cytoplasmic preassembly of different subsets of axonemal dyneins. J Cell Biol 190: 65-71.

Yamamoto R, Song K, Yanagisawa HA, Fox L, Yagi T, Wirschell M, Hirono M, Kamiya R, Nicastro D, Sale WS. 2013. The MIA complex is a conserved and novel dynein regulator essential for normal ciliary motility. J Cell Biol 201: 263-278.

Yanagisawa HA, Kamiya R. 2001. Association between actin and light chains in Chlamydomonas flagellar inner-arm dyneins. Biochem Biophys Res Commun 288: 443-447.

Yanagisawa HA, Kamiya R. 2004. A tektin homologue is decreased in Chlamydomonas mutants lacking an axonemal inner-arm dynein. Mol Biol Cell 15: 2105-2115.

Yang P, Sale WS. 1998. The $M_{\mathrm{r}}$ 140,000 intermediate chain of Chlamydomonas flagellar inner arm dynein is a WD-repeat protein implicated in dynein arm anchoring. Mol Biol Cell 9: 3335-3349.

Yang P, Sale WS. 2000. Casein kinase I is anchored on axonemal doublet microtubules and regulates flagellar dynein phosphorylation and activity. J Biol Chem 275: 18905-18912.

Yang P, Diener DR, Rosenbaum JL, Sale WS. 2001. Localization of calmodulin and dynein light chain LC8 in flagellar radial spokes. J Cell Biol 153: 1315-1326.

Yang P, Diener DR, Yang C, Kohno T, Pazour GJ, Dienes JM, Agrin NS, King SM, Sale WS, Kamiya R, et al. 2006. Radial spoke proteins of Chlamydomonas flagella. J Cell Sci 119: 1165-1174.

Yang P, Yang C, Wirschell M, Davis S. 2009. Novel LC8 mutations have disparate effects on the assembly and stability of flagellar complexes. $J$ Biol Chem 284: 31412-31421.

Yang Y, Cochran DA, Gargano MD, King I, Samhat NK, Burger BP, Sabourin KR, Hou Y, Awata J, Parry DA, et al. 2011. Regulation of flagellar motility by the conserved flagellar protein CG34110/ Ccdc135/FAP50. Mol Biol Cell 22: 976-987.

Zhang D, Aravind L. 2012. Novel transglutaminase-like peptidase and C2 domains elucidate the structure, biogenesis and evolution of the ciliary compartment. Cell Cycle 11: 3861-3875. 


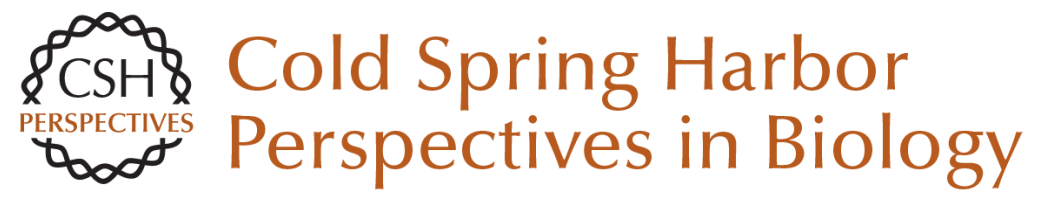

\section{Ciliary Motility: Regulation of Axonemal Dynein Motors}

Rasagnya Viswanadha, Winfield S. Sale and Mary E. Porter

Cold Spring Harb Perspect Biol 2017; doi: 10.1101/cshperspect.a018325

Subject Collection The Cytoskeleton

Microtubules and Microtubule-Associated

Proteins

Holly V. Goodson and Erin M. Jonasson

Motor Proteins

H. Lee Sweeney and Erika L.F. Holzbaur

Myosin-Driven Intracellular Transport Margaret A. Titus

The Actin Cytoskeleton and Actin-Based Motility Tatyana Svitkina

\section{Mechanical Properties of the Cytoskeleton and Cells \\ Adrian F. Pegoraro, Paul Janmey and David A.} Weitz

Intermediate Filaments and the Regulation of Cell Motility during Regeneration and Wound Healing Fang Cheng and John E. Eriksson

Intermediate Filaments and the Plasma Membrane Jonathan C.R. Jones, Chen Yuan Kam, Robert M. Harmon, et al.

Intracellular Motility of Intermediate Filaments Rudolf E. Leube, Marcin Moch and Reinhard Windoffer

\section{Overview of the Cytoskeleton from an}

Evolutionary Perspective

Thomas D. Pollard and Robert D. Goldman

Types I and II Keratin Intermediate Filaments Justin T. Jacob, Pierre A. Coulombe, Raymond Kwan, et al.

Muscle Contraction

H. Lee Sweeney and David W. Hammers

Type III Intermediate Filaments Desmin, Glial

Fibrillary Acidic Protein (GFAP), Vimentin, and

Peripherin

Elly M. Hol and Yassemi Capetanaki

Cytokinesis in Metazoa and Fungi Michael Glotzer

Ciliary Motility: Regulation of Axonemal Dynein Motors

Rasagnya Viswanadha, Winfield S. Sale and Mary

E. Porter

Actin-Based Adhesion Modules Mediate Cell Interactions with the Extracellular Matrix and Neighboring Cells

Alexia I. Bachir, Alan Rick Horwitz, W. James Nelson, et al.

Microtubule-Based Transport and the Distribution,

Tethering, and Organization of Organelles Kari Barlan and Vladimir I. Gelfand

For additional articles in this collection, see http://cshperspectives.cshlp.org/cgi/collection/

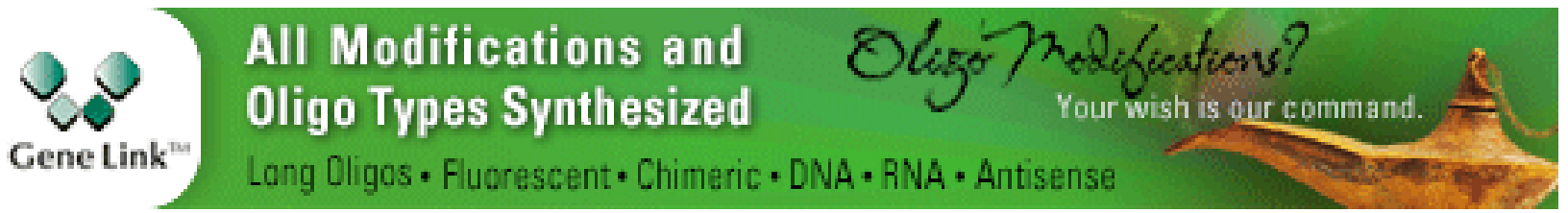

Copyright @ 2017 Cold Spring Harbor Laboratory Press; all rights reserved 
For additional articles in this collection, see http://cshperspectives.cshlp.org/cgi/collection/

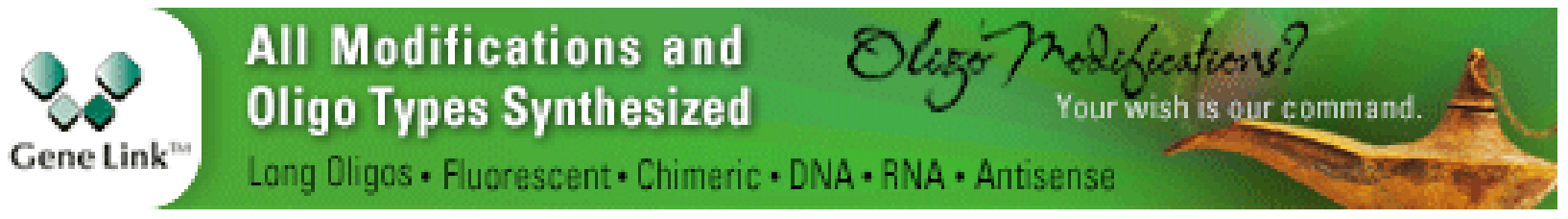

Copyright @ 2017 Cold Spring Harbor Laboratory Press; all rights reserved 